\title{
CLASSIFICATION OF GRAPH $C^{*}$-ALGEBRAS WITH NO MORE THAN FOUR PRIMITIVE IDEALS
}

\author{
SØREN EILERS, GUNNAR RESTORFF, AND EFREN RUIZ
}

\begin{abstract}
We describe the status quo of the classification problem of graph $C^{*}$-algebras with four primitive ideals or less.
\end{abstract}

\section{INTRODUCTION}

The class of graph $C^{*}$-algebras (cf. [Rae05] and the references therein) has proven to be an important and interesting venue for classification theory by $K$-theoretical invariants; in particular with respect to $C^{*}$-algebras with finitely many ideals, and in 2009, the authors formulated the following working conjecture:

Conjecture 1.1. Graph $C^{*}$-algebras $C^{*}(E)$ with finitely many ideals are classified up to stable isomorphism by their filtered, ordered $K$-theory $\mathrm{FK}_{\operatorname{Prim}\left(C^{*}(E)\right)}^{+}\left(C^{*}(E)\right)$.

Here, the filtered, ordered $K$-theory is simply the collection of all $K_{0^{-}}$and $K_{1^{-}}$groups of subquotients of the $C^{*}$-algebra in question, taking into account all the natural transformations among them (details will be given below). The conjecture addresses the possibility of a classification result which is not strong (cf. [Ell10]) in the sense that we do not expect every possible isomorphism at the level of the invariant to lift to the $C^{*}$-algebras.

The conjecture remains open and we are forthwith optimistic about its veracity, although some of the results which have been obtained, as we shall see, seem to indicate that an added condition of finitely generated $K$-theory could be needed. In the present paper we will discuss the status of this conjecture for graph algebras with four or fewer primitive ideals; if the number is three or fewer we can present a complete classification under the condition of finitely generated $K$-theory, but for the number four there are many cases still eluding our methods. Adding, in some cases, the condition of finitely generated $K$-theory - or even stronger, that the graph algebra is unital - we may solve 103 of the 125 cases, leaving less than one fifth of the cases open. Our main contribution in the present paper concerns the class of fan spaces which has not been accessible through the methods we have used earlier, but we will also go through those results in our two papers ERRa] and ERR09] which apply here.

1.1. Tempered primitive ideal spaces. Invoking an idea from [ERS11] we organize our overview using a tempered ideal space of the $C^{*}$-algebra in question. This is defined for any $C^{*}$-algebra with only finitely many ideals as the pair $(\operatorname{Prim}(\mathfrak{A}), \tau)$

Date: September 5, 2018. 
where $\tau: \operatorname{Prim}(\mathfrak{A}) \rightarrow\{0,1\}$ is defined as

$$
\tau(\mathfrak{I})= \begin{cases}0 & K_{0}\left(\mathfrak{I} / \mathfrak{I}_{0}\right)_{+} \neq K_{0}\left(\mathfrak{I} / \mathfrak{I}_{0}\right) \\ 1 & K_{0}\left(\mathfrak{I} / \mathfrak{I}_{0}\right)_{+}=K_{0}\left(\mathfrak{I} / \mathfrak{I}_{0}\right)\end{cases}
$$

with $\mathfrak{I}_{0}$ the maximal proper ideal of $\mathfrak{I}$ (this exists by the fact that $\mathfrak{I}$ is prime and contains only finitely many ideals). We set

$$
X_{\square}=\{x \in X \mid \tau(x)=0\} \quad X_{\mathbf{\square}}=\{x \in X \mid \tau(x)=1\}
$$

To be able to work systematically with these objects, we now give them a combinatorial description.

Definition 1.2. Let $\mathfrak{A}$ be a $C^{*}$-algebra. We let $\operatorname{Prim}(\mathfrak{A})$ denote the primitive ideal space of $\mathfrak{A}$, equipped with the usual hull-kernel topology, also called the Jacobson topology. We always identify the open sets of $\operatorname{Prim}(\mathfrak{A}), \mathbb{O}(\operatorname{Prim}(\mathfrak{A}))$, and the lattice of ideals of $\mathfrak{A}, \mathbb{I}(\mathfrak{A})$, using the lattice isomorphism

$$
U \mapsto \bigcap_{\mathfrak{p} \in \operatorname{Prim}(\mathfrak{A}) \backslash U} \mathfrak{p} .
$$

When $U$ is an open set we write $\mathfrak{A}(U)$ for the corresponding ideal of $\mathfrak{A}$. When $U \supset V$ are both open, so that $U \backslash V$ is locally closed, we write $\mathfrak{A}(U \backslash V)$ for the subquotient $\mathfrak{A}(U) / \mathfrak{A}(V)$.

Note that whenever $X_{\square}$ or $X_{\mathbf{\square}}$ are locally closed, standard results in graph $C^{*}$ algebra theory give that $\mathfrak{A}\left(X_{\square}\right)$ and $\mathfrak{A}\left(X_{\square}\right)$ are $A F$ algebras and $\mathcal{O}_{\infty}$-absorbing algebras, respectively.

Definition 1.3. Let $X$ be a topological space. The specialisation preorder $\prec$ on $X$ is defined by $x \prec y$ if and only if $x \in \overline{\{y\}}$.

A topological space satisfies the $T_{0}$ separation axiom if and only if its specialisation preorder is a partial order.

Definition 1.4. A subset $H$ of a preordered set $(X, \leq)$ is called hereditary if $x \leq y \in H$ implies $x \in H$.

Definition 1.5. Let $(X, \leq)$ be a preordered set. The Alexandrov topology of $X$ is the topology with the closed sets being the hereditary sets.

A topological set is called an Alexandrov space if it carries the Alexandrov topology of some preordered set. The preorder is necessarily the specialisation preorder. A topological space is an Alexandrov space if and only if arbitrary intersections of open sets are open.

Since we are dealing with $C^{*}$-algebras with finite primitive ideal spaces, these are all Alexandrov spaces satisfying the $T_{0}$ separation axiom. Consequently, we can equivalently consider all partial orders on finite sets. The tempered primitive ideal space for a $C^{*}$-algebra with $n$ primitive ideals may hence be uniquely described using a partial order on $\{1, \ldots, n\}$ and a map in $\{0,1\}^{\{1, \ldots, n\}}$.

The transitive reduction of a relation $R$ on a set $X$ is a minimal relation $S$ on $X$ having the same transitive closure as $R$. In general neither existence nor uniqueness are guaranteed, but if the transitive closure of $R$ is antisymmetric and finite, there is a unique transitive reduction. We will illustrate our (finite) topological spaces with graphs of the transitive reduction of the specialisation order, where we write 
an arrow $x \rightarrow y$ if and only if $x$ is less than $y$ in the transitive reduction of the specialisation order (similar to the Hasse diagram). The value of $\tau$ will be indicated by colors of the vertices of the graph; white for 0 and black for 1 .

We obtain a unique signature for each tempered ideal space as follows. Consider the adjacency matrix of the graph of the specialisation order and recall that (by transitivity and antisymmetry) we can always permute the vertices so that the adjacency matrix becomes an upper triangular matrix. Since the relation is reflexive, we will have ones in the diagonal, so without loss of information we may write the values of $\tau$ there. To each such upper triangular matrix

$$
A=\left[\begin{array}{ccccc}
t_{1} & a_{1,2} & & a_{1, n-1} & a_{1, n} \\
& t_{2} & a_{2,3} & & a_{2, n} \\
& & \ddots & \ddots & \\
& & & t_{n-1} & a_{n-1, n} \\
& & & & t_{n}
\end{array}\right]
$$

we associate two binary numbers

$$
a=a_{1,2} a_{1,3} \cdots a_{1, n} a_{2,3} a_{2,4} \cdots a_{2, n} \cdots a_{n-1, n}
$$

and

$$
t=t_{1} \cdots t_{n}
$$

In general, there are several such binary numbers associated with a specialisation order by means of permuting the vertices. We choose the order of the vertices to obtain the smallest possible pair $(a, t)$ ordered lexicographically as the unique identifier for this specific tempered ideal structure. In the interest of conserving space we write hexadecimal expansion of the numbers when referring to a certain structure. We write n.a.t and n.a to indicate signatures and tempered signatures, respectively, defined this way (where $\mathrm{n}$ and a are numbers written in decimal expansions and $t$ is a number written in hexadecimal expansion).

If a primitive ideal space is disconnected, we may classify the $C^{*}$-algebras associated to each component individually. We will hence assume throughout that the $C^{*}$-algebras have connected primitive ideal space (when considering graph algebras, a necessary, but not sufficient, condition for this is that the underlying graphs are connected considered as undirected graphs). Determining the number of connected $T_{0}$-spaces with $n$ points is hard for most $n$; the number has been computed up to $n=16$ in BM02. But for small $n$ even the number of tempered ideal spaces can readily be found by naive enumeration, by first counting all spaces and then performing inverse Euler transform to obtain those that are connected:

\begin{tabular}{|l||c|c|c|c|c|c|}
\hline $\mid$ Prim $(\mathfrak{A}) \mid$ & 1 & 2 & 3 & 4 & 5 & 6 \\
\hline \hline Number of spaces & 1 & 2 & 5 & 16 & 63 & 318 \\
\hline Number of connected spaces & 1 & 1 & 3 & 10 & 44 & 238 \\
\hline \hline Number of tempered spaces & 2 & 10 & 62 & 510 & 5292 & 69364 \\
\hline Number of connected tempered spaces & 2 & 4 & 20 & 125 & 1058 & 11549 \\
\hline
\end{tabular}

We will restrict our attention to $|\operatorname{Prim}(\mathfrak{A})| \leq 4$ and hence have 15 (connected) primitive ideal space 1 which may be given temperatures in a total of 151 different ways to concern ourselves with:

\footnotetext{
${ }^{1}$ The space $4 . E$ was forgotten on page 230 of $\mathrm{MNb}$
} 


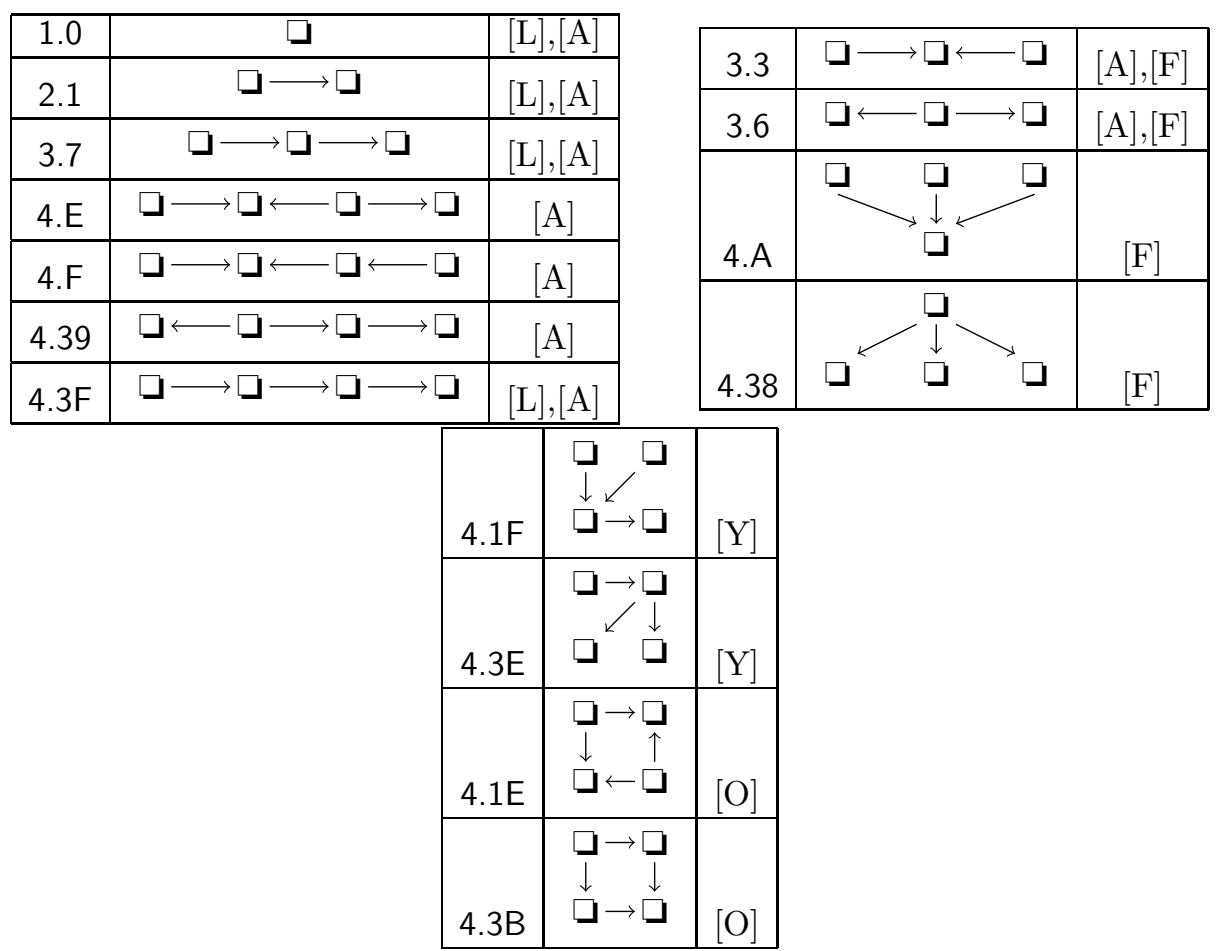

where $\square$ just indicates that it is either $\square$ or

We call a finite $T_{0}$ space linear $([\mathrm{L}])$ if its partial order is total. Following [BK] we call it an accordion space ([A]) if the symmetrization of the space is the symmetrization of a linear space. We call it a fan space $([\mathrm{F}])$ when there is a smallest or largest element in the preorder, so that when this is removed, what remains is a disjoint union of linear spaces. The remaining spaces we organize as [Y]-spaces and $[\mathrm{O}]$-spaces as indicated. In Section 6 below we summarize our results subject to this organization.

1.2. The invariant. Let $\mathfrak{A}$ be a $C^{*}$-algebra with finitely many ideals and set $\mathrm{X}=$ $\operatorname{Prim}(\mathfrak{A})$. Note that for any locally closed subset $Y=U \backslash V$ of $X$, we have two groups $K_{0}(\mathfrak{A}(Y))$ and $K_{1}(\mathfrak{A}(Y))$. Moreover, for any three open subsets $U \subseteq V \subseteq W$ of $X$, we have a six term exact sequence

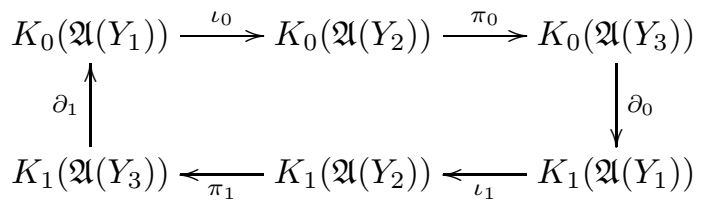

where $Y_{1}=V \backslash U, Y_{2}=W \backslash U$, and $Y_{3}=W \backslash V$. The filtered, ordered $K$ theory $\mathrm{FK}_{\mathbf{X}}^{+}(\mathfrak{A})$ of $\mathfrak{A}$ is the collection of all $K$-groups thus occurring, equipped with order on $K_{0}$ and the natural transformations $\left\{\iota_{*}, \pi_{*}, \partial_{*}\right\}$.

Consequently, if also $\operatorname{Prim}(\mathfrak{B})=X$, we write $\mathrm{FK}_{X}^{+}(\mathfrak{A}) \cong \mathrm{FK}_{X}^{+}(\mathfrak{B})$ if for each locally closed subset $Y$ of $X$, there exist group isomorphisms

$$
\alpha_{*}^{Y}: K_{*}(\mathfrak{A}(Y)) \rightarrow K_{*}(B(Y))
$$


preserving all natural transformations in such a way that all $\alpha_{0}^{Y}$ are also order isomorphisms. All components of this invariant are readily computable ([CET]), and often, much of it is redundant. We will not pursue that issue here.

The filtered $K$-theory $\mathrm{FK}_{\mathbf{X}}(\mathfrak{A})$ of $\mathfrak{A}$ is defined analogously by disregarding the order structure on $K_{0}$. The filtered (ordered) $K$-theory over a finite $T_{0}$-space $X$ can also be used for $C^{*}$-algebras over $X$ without being tight 2

1.3. Graph $C^{*}$-algebra. A graph $\left(E^{0}, E^{1}, r, s\right)$ consists of a countable set $E^{0}$ of vertices, a countable set $E^{1}$ of edges, and maps $r: E^{1} \rightarrow E^{0}$ and $s: E^{1} \rightarrow E^{0}$ identifying the range and source of each edge. If $E$ is a graph, the graph $C^{*}$-algebra $C^{*}(E)$ is the universal $C^{*}$-algebra generated by mutually orthogonal projections $\left\{p_{v}: v \in E^{0}\right\}$ and partial isometries $\left\{s_{e}: e \in E^{1}\right\}$ with mutually orthogonal ranges satisfying

(1) $s_{e}^{*} s_{e}=p_{r(e)} \quad$ for all $e \in E^{1}$

(2) $s_{e} s_{e}^{*} \leq p_{s(e)} \quad$ for all $e \in E^{1}$

(3) $p_{v}=\sum_{\left\{e \in E^{1}: s(e)=v\right\}} s_{e} s_{e}^{*} \quad$ for all $v$ with $0<\left|s^{-1}(v)\right|<\infty$.

The countability hypothesis ensures that all our graph $C^{*}$-algebras are separable, which is a necessary hypothesis for many of the classification results. We will be mainly interested in graph $C^{*}$-algebras with real rank zero. For a graph $E$, we have that the real rank of $C^{*}(E)$ is zero if and only if $E$ is satisfying Condition (K), i.e., no vertex of $E$ is the base point of exactly one simple cycle (see Theorem 3.5 of [JJ]). Moreover, by Proposition 3.3 of [JJ], every graph $C^{*}$-algebra with finitely many ideals has real rank zero. Thus, every graph $C^{*}$-algebra with finitely many ideals has a norm-full projection, and by Bro77, every graph $C^{*}$-algebra with finitely many ideals is stably isomorphic to a unital $C^{*}$-algebra.

Throughout the paper we will use the following facts about graph $C^{*}$-algebras without further mention.

Theorem 1.6. Let $C^{*}(E)$ be a unital graph $C^{*}$-algebra satisfying Condition (K).

(1) Every ideal of $C^{*}(E)$ is stably isomorphic to a unital graph $C^{*}$-algebra.

(2) Every sub-quotient of $C^{*}(E)$ is stably isomorphic to a unital graph $C^{*}$-algebra.

(3) The K-groups of every sub-quotient of $C^{*}(E)$ is finitely generated.

(4) Every non-unital simple sub-quotient of $C^{*}(E)$ that is an AF-algebra is isomorphic to $\mathbb{K}$.

Proof. As in the proof of Theorem 5.7 (4) of [MT07] (see also BHRS, Proposition 3.4]), every ideal of a graph $C^{*}$-algebra satisfying Condition $(\mathrm{K})$ is Morita equivalent to $C^{*}(F)$, where $F^{0} \subseteq E^{0}$. Hence, (1) holds since a graph $C^{*}$-algebra $C^{*}(E)$ is unital if and only if $E^{0}$ is finite. (2) follows from (1) and BHRS, Corollary 3.5]. (3) follows from (2) and DT02, Theorem 3.1].

Suppose $C^{*}(F)$ is a simple unital AF-algebra. Then $F$ has no cycles. Since $C^{*}(F)$ is unital, $F^{0}$ is finite. Therefore, $F$ has a sink. By [DT05, Corollary 2.15], every singular vertex must be reached by any other vertex since $C^{*}(F)$ is simple. Thus, $F$ must be a finite graph. Hence, $C^{*}(F) \cong \mathrm{M}_{n}$. From this observation, (4) follows from (1) and (2) since any non-unital simple $C^{*}$-algebra stably isomorphic to $\mathbb{K}$ is isomorphic to $\mathbb{K}$.

\footnotetext{
${ }^{2}$ Although this is not exactly the same definition as the filtrated $K$-theory in $\mathrm{MNa}$, it is known to be the same for all the cases where we have a UCT. For more on this invariant and $C^{*}$-algebras over $X$ the reader is referred to $\mathrm{MNa}$ and the references therein.
} 
See [Rae05] and the references therein for more on graph $C^{*}$-algebras.

\section{General theOry}

We first describe the situations in which the graph algebras can be classified using widely applicable results.

2.1. The $\boldsymbol{A F}$ case. The $A F$ case corresponds to temperatures that are constantly 0 . We incur these at the tempered signatures 1.0.0, 2.1.0, 3.3.0, 3.6.0, 3.7.0, 4.A.0, 4.E.0, 4.F.0, 4.1E.0, 4.1F.0, 4.38.0, 4.39.0, 4.3B.0, 4.3E.0, and 4.3F.0. Of course the classification question is resolved by Elliott's theorem:

Theorem 2.1 ([Ell76]). AF algebras are classified up to stable isomorphism by their ordered $K_{0}$-group.

2.2. The purely infinite case. Recall that there are three notions of pure infiniteness for non-simple $C^{*}$-algebras, namely pure infiniteness, strong pure infiniteness, and $\mathcal{O}_{\infty}$-absorption, introduced by E. Kirchberg and M. Rørdam; cf. KR00. and KR02.

Corollary 2.2. For each nuclear, separable $C^{*}$-algebra $\mathfrak{A}$ with finite primitive ideal space, the following are equivalent:

(a) $\mathfrak{A}$ is purely infinite,

(b) $\mathfrak{A}$ is strongly purely infinite,

(c) $\mathfrak{A}$ is $\mathcal{O}_{\infty}$-absorbing, i.e., $\mathfrak{A} \otimes \mathcal{O}_{\infty} \cong \mathfrak{A}$.

Proof. It follows from Theorem 9.1 and Corollary 9.2 of KR02 that (c) implies (b), that (b) implies (a), and that the three coincide in the simple case. It follows from Proposition 3.5 of [KR02, that pure infiniteness passes to ideals and subquotients. Thus it follows from [TW07] that (a) implies (c).

The purely infinite case (the $\mathcal{O}_{\infty}$-absorbing case) corresponds to temperatures that are constantly 1 . We incur these at the tempered signatures 1.0.1, 2.1.3, 3.3.7, 3.6.7, 3.7.7, 4.A.F, 4.E.F, 4.F.F, 4.1E.F, 4.1F.F, 4.38.F, 4.39.F, 4.3B.F, 4.3E.F, and 4.3F.F. As we will outline below, all but the case 4.1E.F are resolved through the recent work of many hands.

The isomorphism result of Kirchberg (cf. [Kir94 and Kir00]) reduces the classification problem of nuclear and strongly purely infinite $C^{*}$-algebras which are also in the bootstrap class to an isomorphism problem in ideal-related $K K$-theory. Since all purely infinite graph $C^{*}$-algebras fall in this class we may hence confirm Conjecture 1.1 in the purely infinite case by providing a universal coefficient theorem which allows the lifting of isomorphisms at the level of filtered $K$-theory to invertible $K K_{X}$-classes. This, however, is not known to be possible in general. Indeed, Meyer and Nest in $\mathrm{MNa}$, showed that there are purely infinite $C^{*}$-algebras over the space 4.A which fails to have this property, but since the examples provided there cannot possibly come from graph algebras, the question remains open in general. The work of Bentmann and Köhler established that general UCTs are available precisely when the space $X$ is an accordion space, and Arklint with the second and third named authors provided UCTs for other spaces, including 4.A, under the added assumption that the $C^{*}$-algebra has real rank zero which is automatic here. Specializing even further, Arklint, Bentmann and Katsura provided a UCT which applies for our space 4.3B under the added assumption that the $C^{*}$-algebra has 
real rank zero and that the $K_{1}$ groups of all subquotients are free, which also is automatic here. The space 4.1E remains open. In conclusion:

Theorem 2.3. Purely infinite, separable, nuclear $C^{*}$-algebras $\mathfrak{A}$ with finite primitive ideal space $X$ in the bootstrap class of Meyer and Nest (i.e., all simple subquotients are in the bootstrap class of Rosenberg and Schochet) are classified up to stable isomorphism by their filtered $K$-theory $\mathrm{FK}_{X}(-)$ in the cases

(i) $X$ is an accordion space $[1.0,2.1,3.3,3.6,3.7,4 . E, 4 . F, 4.39,4.3 F]$ (Kir94, [NCP00, Ror97, Res08, $\mathrm{MNa}, \mathrm{BK}$, Kir00)

(ii) $X$ is one of the spaces $4 . A, 4.38,4.1 F, 4.3 E$ and $\operatorname{rr}(\mathfrak{A})=0$. (ARR12)

(iii) $X$ is the space $4.3 B, \operatorname{rr}(\mathfrak{A})=0$, and $K_{1}(\mathfrak{J} / \mathfrak{I})$ is free for any $\mathfrak{I} \triangleleft \mathfrak{J} \unlhd \mathfrak{A}$ ( $\mathrm{ABK}$ )

2.3. The separated case. The classification problem for the two mixed cases with $|\operatorname{Prim}(\mathfrak{A})|=2$ not covered by the results mentioned above - the tempered signatures 2.1.1 and 2.1.2 - were resolved in ET10] drawing heavily on ERR09. In [ERRa, we generalized this to more complicated cases having the separation property which is automatic in the two-point case, as detailed below. The idea is to find an ideal $\mathfrak{I}$ such that $\mathfrak{I}$ is $A F$ and $\mathfrak{A} / \mathfrak{I}$ is $\mathcal{O}_{\infty}$-absorbing, or vice versa. We do not know in general how to prove classification in this case, but under certain added assumptions related to the notion of fullness, this leads to results that may be used to resolve the cases of tempered signature 3.7.1, 3.7.3, 4.F.1, 4.1F.1, 4.1F.3, 4.3B.1, 4.3F.1, 4.3F.3, 4.3F.7 by Proposition 2.5 below and 3.7.4, 3.7.6, 4.39.8, 4.3B.8, 4.3E.8, 4.3E.C, 4.3F.8, 4.3F.C, 4.3F.E by Proposition 2.6.

Definition 2.4. Let $n>1$ be a given integer. Then we let $\mathrm{X}_{n}$ denote the partially ordered set (actually totally ordered) $\mathrm{X}_{n}=\{1,2, \ldots, n\}$ with the usual order. For $a, b \in \mathrm{X}_{n}$ with $a \leq b$, we let $[a, b]$ denote the set $\left\{x \in \mathrm{X}_{n}: a \leq x \leq b\right\}$.

Proposition 2.5. Let $\mathfrak{A}_{1}$ and $\mathfrak{A}_{2}$ be separable, nuclear, $C^{*}$-algebras over $\mathrm{X}_{n}$ in the bootstrap class of Meyer and Nest (i.e., every simple subquotient is in the bootstrap class of Rosenberg and Schochet). Suppose $\mathfrak{A}_{i}(\{1\})$ is an AF algebra and $\mathfrak{A}_{i}([2, n])$ is a tight stable $\mathcal{O}_{\infty}$-absorbing $C^{*}$-algebra over $[2, n]$, and $\mathfrak{A}_{i}(\{2\})$ is an essential ideal of $\mathfrak{A}_{i}([1,2])$. Then $\mathfrak{A}_{1} \otimes \mathbb{K} \cong \mathfrak{A}_{2} \otimes \mathbb{K}$ if and only if there exists an isomorphism $\alpha: \mathrm{FK}_{\mathrm{X}_{n}}\left(\mathfrak{A}_{1}\right) \rightarrow \mathrm{FK}_{\mathrm{X}_{n}}\left(\mathfrak{A}_{2}\right)$ such that $\alpha_{\{1\}}$ is positive.

Proposition 2.6. Let $\mathfrak{A}_{1}$ and $\mathfrak{A}_{2}$ be graph $C^{*}$-algebras satisfying Condition (K). Suppose $\mathfrak{A}_{i}$ is a $C^{*}$-algebra over $\mathbf{X}_{n}$ such that $\mathfrak{A}_{i}(\{n\})$ is an $A F$ algebra, for every ideal $\mathfrak{I}$ of $\mathfrak{A}_{i}$ we have that $\mathfrak{I} \subseteq \mathfrak{A}_{i}(\{n\})$ or $\mathfrak{A}_{i}(\{n\}) \subseteq \mathfrak{I}$, and $\mathfrak{A}_{i}([1, n-1])$ is a tight, $\mathcal{O}_{\infty}$-absorbing $C^{*}$-algebra over $[1, n-1]$. Then $\mathfrak{A}_{1} \otimes \mathbb{K} \cong \mathfrak{A}_{2} \otimes \mathbb{K}$ if and only if there exists an isomorphism $\alpha: \mathrm{FK}_{\mathbf{X}_{n}}\left(\mathfrak{A}_{1}\right) \rightarrow \mathrm{FK}_{\mathbf{X}_{n}}\left(\mathfrak{A}_{2}\right)$ such that $\alpha_{\{n\}}$ is positive.

\section{FAN SPACES}

In this section, we develop methods to deal mainly with the spaces 3.3, 3.6, 4.A, 4.38. We observe the following in ERRa.

Lemma 3.1. Let $E$ be a graph such that $C^{*}(E)$ has finitely many ideals and assume that $\mathfrak{I} \triangleleft \mathfrak{J} \unlhd C^{*}(E)$ are ideals. Then

(i) $C^{*}(E) \otimes \mathbb{K}$ has the corona factorization property

(ii) $(\mathfrak{J} / \mathfrak{I}) \otimes \mathbb{K}$ is of the form $C^{*}(F) \otimes \mathbb{K}$ for some graph $F$

(iii) $(\mathfrak{J} / \mathfrak{I}) \otimes \mathbb{K}$ has the corona factorization property 
The graph $F$ above can be chosen as a subgraph of the Drinen-Tomforde desingularization of $E$ ([DT05]).

Definition 3.2. For each $C^{*}$-algebra $\mathfrak{A}$, we let $\mathcal{M}(\mathfrak{A})$ and $\mathcal{Q}(\mathfrak{A})$ denote the multiplier algebra and the corona algebra of $\mathfrak{A}$, respectively.

For each extension

$$
\mathfrak{e}: 0 \rightarrow \mathfrak{B} \rightarrow \mathfrak{E} \rightarrow \mathfrak{A} \rightarrow 0,
$$

we let $\eta_{\mathfrak{e}}: \mathfrak{A} \rightarrow \mathcal{Q}(\mathfrak{B})$ denote the Busby map of the extension.

Moreover, for each surjective (or, more generally, proper) $*$-homomorphism $\varphi$ : $\mathfrak{A} \rightarrow \mathfrak{B}$, we let $\widetilde{\varphi}: \mathcal{M}(\mathfrak{A}) \rightarrow \mathcal{M}(\mathfrak{B})$ and $\bar{\varphi}: \mathcal{Q}(\mathfrak{A}) \rightarrow \mathcal{Q}(\mathfrak{B})$ denote the unique extension to the multiplier algebras and the induced $*$-homomorphism between the corona algebras, respectively (cf. $§ 2.1$ of [ELP99]).

Lemma 3.3. Let $\left(\mathfrak{B}_{i}\right)_{i \in I}$ be a family of $C^{*}$-algebras (small enough for direct sums and products to exist). Let $\pi_{j}: \bigoplus_{i \in I} \mathfrak{B}_{i} \rightarrow \mathfrak{B}_{j}$ denote the canonical projection, for each $j \in I$. Then there is a canonical isomorphism $\prod_{i \in I} \tilde{\pi}_{i}: \mathcal{M}\left(\bigoplus_{i \in I} \mathfrak{B}_{i}\right) \rightarrow$ $\prod_{i \in I} \mathcal{M}\left(\mathfrak{B}_{i}\right)$ which has the unique extension $\tilde{\pi}_{j}: \mathcal{M}\left(\bigoplus_{i \in I} \mathfrak{B}_{i}\right) \rightarrow \mathcal{M}\left(\mathfrak{B}_{j}\right)$ of $\pi_{j}$ as the $j$ 'th coordinate map.

Consequently, if $I$ is finite, there is an induced isomorphism $\prod_{i \in I} \overline{\pi_{i}}: \mathcal{Q}\left(\bigoplus_{i \in I} \mathfrak{B}_{i}\right) \rightarrow$ $\prod_{i \in I} \mathcal{Q}\left(\mathfrak{B}_{i}\right)$, and it induces homomorphisms $\overline{\pi_{j}}: \mathcal{Q}\left(\bigoplus_{i \in I} \mathfrak{B}_{i}\right) \rightarrow \mathcal{Q}\left(\mathfrak{B}_{j}\right)$ as the $j$ 'th coordinate map. In this case, the direct product coincides with the direct sum.

Proof. Here we view the multiplier algebras as the algebras of double centralisers (cf. pp. 39 and 81-82 in [Mur90]). Let $\left(\rho_{1}, \rho_{2}\right)$ be a double centralizer on $\bigoplus_{i \in I} \mathfrak{B}_{i}$ (i.e., an arbitrary element of $\mathcal{M}\left(\bigoplus_{i \in I} \mathfrak{B}_{i}\right)$ ). Using an approximate unit, it is easy to see that $\rho_{1}$ and $\rho_{2}$ restricted to $\mathfrak{B}_{j}$ map into $\mathfrak{B}_{j}$ itself. In this way we get a canonical *-homomorphism from $\mathcal{M}\left(\bigoplus_{i \in I} \mathfrak{B}_{i}\right)$ to $\mathcal{M}\left(\mathfrak{B}_{j}\right)$. By the universal property of the direct product, we get a $*$-homomorphism $\varphi$ from $\mathcal{M}\left(\bigoplus_{i \in I} \mathfrak{B}_{i}\right)$ to $\prod_{i \in I} \mathcal{M}\left(\mathfrak{B}_{i}\right)$, where the $j$ 'th coordinate map clearly is an extension of $\pi_{j}$ to the multiplier algebras, and hence it is the extension $\widetilde{\pi_{j}}$ of $\pi_{j}$. Clearly, $\varphi$ is injective. It is also easy to show that $\varphi$ is surjective by constructing the preimage.

Therefore, if $I$ is finite, the direct product of the short exact sequences

$$
0 \longrightarrow \mathfrak{B}_{j} \longrightarrow \mathcal{M}\left(\mathfrak{B}_{j}\right) \longrightarrow \mathcal{Q}\left(\mathfrak{B}_{j}\right) \longrightarrow 0
$$

is canonically isomorphic to

$$
0 \longrightarrow \bigoplus_{i \in I} \mathfrak{B}_{i} \longrightarrow \mathcal{M}\left(\bigoplus_{i \in I} \mathfrak{B}_{i}\right) \longrightarrow \mathcal{Q}\left(\bigoplus_{i \in I} \mathfrak{B}_{i}\right) \longrightarrow 0
$$

\subsection{Primitive ideal space with $n$ maximal elements.}

Assumption 3.4. For this subsection, let $n>1$ be a fixed integer, and let $X_{i}=\mathrm{X}_{l_{i}}$ for $i=1,2, \ldots, n$, where $l_{1}, l_{2}, \ldots, l_{n}$ are fixed positive integers. Let, moreover,

$$
X=\{m\} \sqcup X_{1} \sqcup X_{2} \sqcup \cdots \sqcup X_{n}
$$

and define a partial order on $X$ as follows. The element $m$ is the least element of $X$, and for each $i=1,2, \ldots, n$, if $x, y \in X_{i}$ then $x \leq y$ in $X$ if and only if $x \leq y$ in $X_{i}$. No other relations exist between the elements of $X$. 
Lemma 3.5. Let $\mathfrak{A}$ be a tight $C^{*}$-algebra over $X$ and let $k \in\{1,2, \ldots, n\}$ be given. Consider the extensions

$$
\mathfrak{e}: 0 \rightarrow \mathfrak{A}(X \backslash\{m\}) \rightarrow \mathfrak{A} \rightarrow \mathfrak{A}(\{m\}) \rightarrow 0
$$

and

$$
\mathfrak{e} \cdot \pi_{k}: 0 \rightarrow \mathfrak{A}\left(X_{k}\right) \rightarrow \mathfrak{A}\left(X_{k} \cup\{m\}\right) \rightarrow \mathfrak{A}(\{m\}) \rightarrow 0,
$$

where $\pi_{k}: \mathfrak{A}(X \backslash\{m\}) \rightarrow \mathfrak{A}\left(X_{k}\right)$ is the canonical quotient $*$-homomorphism.

Then $\eta_{\mathfrak{e} \cdot \pi_{k}}=\bar{\pi}_{k} \circ \eta_{\mathfrak{e}}$, and $\bar{\pi}_{k} \circ \eta_{\mathfrak{e}}$ is injective.

Proof. Note that the diagram

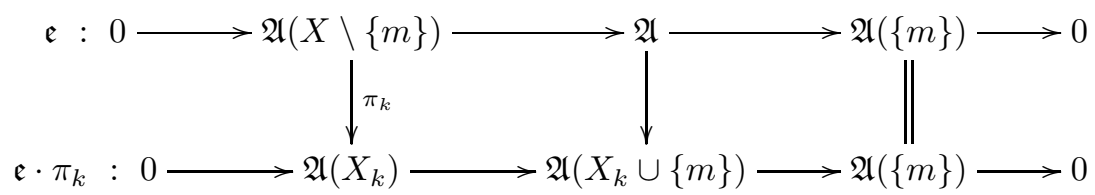

is commutative. Since $\pi_{k}$ is surjective, by Theorem 2.2 of [ELP99, $\bar{\pi}_{k} \circ \eta_{\mathfrak{e}}=\eta_{\mathfrak{e} \cdot \pi_{k}}$. Also note, that Corollary 4.3 of [ELP99] justifies the notation $\mathfrak{e} \cdot \pi_{k}$. Suppose $\bar{\pi}_{k} \circ \eta_{\mathfrak{e}}$ is not injective, then $\bar{\pi}_{k} \circ \eta_{\mathfrak{e}}=0$ since $\mathfrak{A}(\{m\})$ is a simple $C^{*}$-algebra. Hence, $\mathfrak{A}\left(X_{k} \cup\{m\}\right) \cong \mathfrak{A}\left(X_{k}\right) \oplus \mathfrak{A}(\{m\})$. Since $\mathfrak{A}\left(X_{k} \cup\{m\}\right) \cong \mathfrak{A} / \mathfrak{A}\left(X \backslash\left(X_{k} \cup\{m\}\right)\right)$, then there exist proper ideals $\mathfrak{I}$ and $\mathfrak{J}$ of $\mathfrak{A}$ such that $\mathfrak{I}+\mathfrak{J}=\mathfrak{A}$ and $\mathfrak{I} \cap \mathfrak{J}=$ $\mathfrak{A}\left(X \backslash\left(X_{k} \cup\{m\}\right)\right)$. But this contradicts the fact that $\mathfrak{A}$ is a tight $C^{*}$-algebra over $X$. Hence, $\bar{\pi}_{k} \circ \eta_{\mathfrak{e}}$ is injective.

Lemma 3.6. Let $\mathfrak{A}$ be a tight $C^{*}$-algebra over $X$. Then

$$
\mathfrak{e}: 0 \rightarrow \mathfrak{A}(X \backslash\{m\}) \rightarrow \mathfrak{A} \rightarrow \mathfrak{A}(\{m\}) \rightarrow 0
$$

is full if and only if $\mathfrak{e} \cdot \pi_{k}$ is full for all $k=1,2, \ldots, n$.

Proof. By Lemma 3.5 $\eta_{\mathfrak{e} \cdot \pi_{k}}=\bar{\pi}_{k} \circ \eta_{\mathfrak{e}}$. Thus, if $\mathfrak{e}$ is a full extension, then $\mathfrak{e} \cdot \pi_{k}$ is a full extension since $\bar{\pi}_{k}$ is surjective. Suppose $\mathfrak{e} \cdot \pi_{k}$ is a full extension for all $k=1,2, \ldots, n$. Note that $\mathfrak{A}(X \backslash\{m\})$ is $\bigoplus_{j=1}^{n} \mathfrak{A}\left(X_{j}\right)$ and thus from Lemma 3.3 it follows that the $j$ 'th coordinate map of $\left(\bigoplus_{i=1}^{n} \bar{\pi}_{i}\right) \circ \eta_{\mathfrak{e}}$ is exactly $\overline{\pi_{j}} \circ \eta_{\mathfrak{e}}=\eta_{\mathfrak{e} \cdot \pi_{j}}$ (according to Lemma 3.5). Since $\bigoplus_{i=1}^{n} \bar{\pi}_{i}$ is an isomorphism and since $\mathfrak{e} \cdot \pi_{k}$ is a full extension for all $k=1,2, \ldots, n$, we have that $\mathfrak{e}$ is a full extension. That this direct sum of full extensions is again full can easily be shown by first cutting down to each coordinate.

The signatures 3.6.1, 3.6.5, 4.39.1, 4.39.3, 4.39.4, 4.39.5, 4.39.7, 4.38.1, 4.38.3, 4.38.7 are covered by the following theorem.

Theorem 3.7. Let $\mathfrak{A}$ and $\mathfrak{B}$ be graph $C^{*}$-algebras that are tight $C^{*}$-algebras over $X$. Assume that there exists an isomorphism $\alpha: \mathrm{FK}_{X}^{+}(\mathfrak{A}) \rightarrow \mathrm{FK}_{X}^{+}(\mathfrak{B})$. Assume, moreover, that $\mathfrak{A}(\{m\})$ is an $A F$ algebra and that $X_{\square}$ is hereditary. Then $\mathfrak{A} \otimes \mathbb{K} \cong$ $\mathfrak{B} \otimes \mathbb{K}$.

Proof. We may assume that $\mathfrak{A}$ and $\mathfrak{B}$ are stable $C^{*}$-algebras. Note that for each $x \in X, \mathfrak{A}(\{x\})$ is an $A F$ algebra if and only if since $\mathfrak{B}(\{x\})$ is an $A F$ algebra, and $\mathfrak{A}(\{x\})$ is $\mathcal{O}_{\infty}$-absorbing if and only if $\mathfrak{B}(\{x\})$ is $\mathcal{O}_{\infty}$-absorbing (since there exists a positive isomorphism from $K_{0}(\mathfrak{A}(\{x\}))$ to $\left.K_{0}(\mathfrak{B}(\{x\}))\right)$. Specifically, $\mathfrak{B}(\{m\})$ is an $A F$ algebra. First we assume that $X_{\square} \neq \emptyset$ and $X_{\square} \backslash\{m\} \neq \emptyset$.

Note that $\mathfrak{A}\left(X_{\square}\right)$ and $\mathfrak{B}\left(X_{\square}\right)$ are $A F$ algebras. Since $\alpha_{X_{\square}}: K_{0}\left(\mathfrak{A}\left(X_{\square}\right)\right) \rightarrow$ $K_{0}\left(\mathfrak{B}\left(X_{\square}\right)\right)$ is a positive isomorphism, there exists an isomorphism $\beta: \mathfrak{A}\left(X_{\square}\right) \rightarrow$ 
$\mathfrak{B}\left(X_{\square}\right)$ such that $K_{0}(\beta)=\alpha_{X_{\square}}$ (by Elliott's classification result [Ell76]). Since $\mathfrak{A}\left(X_{\square}\right)$ and $\mathfrak{B}\left(X_{\square}\right)$ are $A F$ algebras and $\beta$ is an $X_{\square}$-equivariant isomorphism, we have that $K_{0}\left(\beta_{Y}\right)=\alpha_{Y}$ for all $Y \in \mathbb{L} \mathbb{C}(X)$ such that $Y \subseteq X_{\square}$. In particular, $K_{0}\left(\beta_{\{x\}}\right)=\alpha_{\{x\}}$ for all $x \in X_{\square}$.

Let $X_{\mathbf{\square}}^{\text {min }}$ be the set of minimal elements of $X \mathbf{\square}$, and for each $a, b \in X$ let

$$
\begin{aligned}
{[a, \infty) } & =\{x \in X: a \leq x\}, \\
{[a, b) } & =\{x \in X: a \leq x<b\} .
\end{aligned}
$$

Let $x \in X^{\text {min }}$ be given. Let $i_{x} \in\{1,2, \ldots, n\}$ be the unique number such that $x \in X_{i_{x}}$. Note that $X_{i_{x}} \sqcup\{m\}=[m, x) \cup[x, \infty)$, which we will denote by $\tilde{X}_{i_{x}}$. Let, moreover,

$$
\mathfrak{e}_{x}^{\mathfrak{A}}: 0 \rightarrow \mathfrak{A}([x, \infty)) \rightarrow \mathfrak{A}\left(\widetilde{X}_{i_{x}}\right) \rightarrow \mathfrak{A}([m, x)) \rightarrow 0 .
$$

and

$$
\mathfrak{e}_{x}^{\mathfrak{B}}: 0 \rightarrow \mathfrak{B}([x, \infty)) \rightarrow \mathfrak{B}\left(\widetilde{X}_{i_{x}}\right) \rightarrow \mathfrak{B}([m, x)) \rightarrow 0 .
$$

Since $\alpha: \mathrm{FK}_{X}^{+}(\mathfrak{A}) \rightarrow \mathrm{FK}_{X}^{+}(\mathfrak{B})$ is an isomorphism, we also have an isomorphism $\alpha_{\widetilde{X}_{i_{x}}}: \mathrm{FK}_{\widetilde{X}_{i_{x}}}^{+}\left(\mathfrak{A}\left(\widetilde{X}_{i_{x}}\right)\right) \rightarrow \mathrm{FK}_{\widetilde{X}_{i_{x}}}^{+}\left(\mathfrak{B}\left(\widetilde{X}_{i_{x}}\right)\right)$. So by Theorem 4.14 of $\mathrm{MNa}$, Kirchberg [Kir00, and Theorem 3.3 of [ERRa], there exists an isomorphism $\varphi^{x}$ : $\mathfrak{A}([x, \infty)) \rightarrow \mathfrak{B}([x, \infty))$ such that $K_{*}\left(\varphi^{x}\right)=\alpha_{[x, \infty)}$, and

$$
\left[\eta_{\mathfrak{e}_{x}^{\mathfrak{B}}} \circ \beta_{[m, x)}\right]=\left[\overline{\varphi^{x}} \circ \eta_{\mathfrak{e}_{x}^{\mathfrak{A}}}\right]
$$

in $K K^{1}(\mathfrak{A}([m, x)), \mathfrak{B}([x, \infty)))$, since $K K\left(\beta_{[m, x)}\right)$ is the unique lifting of $\alpha_{[m, x)}$.

As in the proof of Proposition 6.3 of [ERRa, Corollary 5.3 of [ERRa] implies that $\eta_{\mathfrak{e}_{x}^{\mathfrak{A}}}$ and $\eta_{\mathfrak{e}_{x}^{\mathfrak{B}}}$ are full extensions, and thus also the extensions with Busby maps $\eta_{\mathfrak{e}_{x}^{\mathfrak{B}}} \circ \beta_{[m, x)}$ and $\overline{\varphi^{x}} \circ \eta_{\mathfrak{e}_{x}^{\mathfrak{A}}}$ are full. Since the extensions are non-unital and $\mathfrak{B}([x, \infty))$ satisfies the corona factorization property, there exists a unitary $u_{x} \in$ $\mathcal{M}(\mathfrak{B}([x, \infty)))$ such that

$$
\eta_{\mathfrak{e}_{x}^{\mathfrak{B}}} \circ \beta_{[m, x)}=\operatorname{Ad}\left(\overline{u_{x}}\right) \circ \overline{\varphi^{x}} \circ \eta_{\mathfrak{e}_{x}^{\mathfrak{A}}}
$$

where $\overline{u_{x}}$ is the image of $u_{x}$ in the corona algebra (this follows from [EK01] and KN06]). Hence, by Theorem 2.2 of [ELP99, there exists an isomorphism $\eta^{x}$ : $\mathfrak{A}\left(\widetilde{X}_{i_{x}}\right) \rightarrow \mathfrak{B}\left(\widetilde{X}_{i_{x}}\right)$ such that $\left(\operatorname{Ad}\left(\overline{u_{x}}\right) \circ \varphi^{x}, \eta^{x}, \beta_{[m, x)}\right)$ is an isomorphism from $\mathfrak{e}_{x}^{\mathfrak{A}}$ to $\mathfrak{e}_{x}^{\mathfrak{B}}$. Let

and

$$
\mathfrak{e}^{\mathfrak{A}}: 0 \rightarrow \mathfrak{A}(X \backslash\{m\}) \rightarrow \mathfrak{A} \rightarrow \mathfrak{A}(\{m\}) \rightarrow 0,
$$

$\mathfrak{e}^{\mathfrak{B}}: 0 \rightarrow \mathfrak{B}(X \backslash\{m\}) \rightarrow \mathfrak{B} \rightarrow \mathfrak{B}(\{m\}) \rightarrow 0$.

Since $\mathfrak{A}\left(\widetilde{X}_{i_{x}}\right)$ and $\mathfrak{B}\left(\widetilde{X}_{i_{x}}\right)$ have linear ideal lattices, this induces an isomorphism

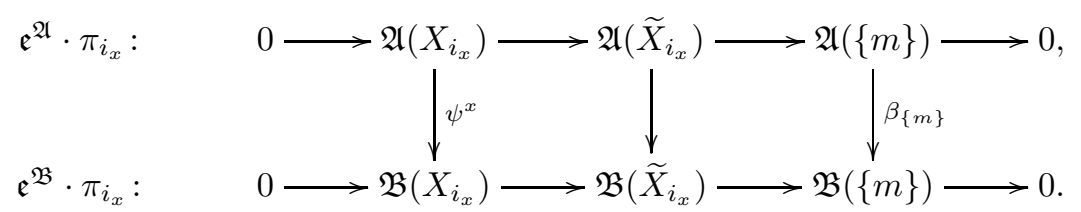

So now by construction,

$$
\overline{\psi^{x}} \circ \eta_{\mathfrak{e}^{\mathfrak{A}} \cdot \pi_{i_{x}}}=\eta_{\mathfrak{e}^{\mathfrak{B}} \cdot \pi_{i_{x}}} \circ \beta_{\{m\}},
$$

for all $x \in X_{\text {min }}^{\text {mand }}$, and

$$
\overline{\beta_{X_{j}}} \circ \eta_{\mathfrak{e}^{\mathfrak{A}} \cdot \pi_{j}}=\eta_{\mathfrak{e}^{\mathfrak{B}} \cdot \pi_{j}} \circ \beta_{\{m\}},
$$


for all $j=1,2, \ldots, n$ satisfying that $\mathfrak{A}\left(X_{j}\right)$ is an $A F$ algebra. Now we define an isomorphism $\theta$ from $\mathfrak{A}(X \backslash\{m\})$ to $\mathfrak{B}(X \backslash\{m\})$ as the direct sum of the $\psi^{x}$ 's and $\beta_{X_{j}}$ 's. We get that (from Lemma 3.3 and Lemma 3.5)

$$
\begin{gathered}
\bar{\theta} \circ \eta_{\mathfrak{e}^{\mathfrak{A}}}=\bar{\theta} \circ\left(\bigoplus_{j=1}^{n} \eta_{\mathfrak{e}^{\mathfrak{A}} \cdot \pi_{j}}\right)=\bigoplus_{j=1}^{n} \overline{\theta_{j}} \circ \eta_{\mathfrak{e}^{\mathfrak{A}} \cdot \pi_{j}}= \\
\bigoplus_{j=1}^{n} \eta_{\mathfrak{e}^{\mathfrak{B}} \cdot \pi_{j}} \circ \beta_{\{m\}}=\left(\bigoplus_{j=1}^{n} \eta_{\mathfrak{e}^{\mathfrak{B}} \cdot \pi_{j}}\right) \circ \beta_{\{m\}}=\eta_{\mathfrak{e}^{\mathfrak{B}}} \circ \beta_{\{m\}},
\end{gathered}
$$

where the $\theta_{j}$ 's denote the corresponding $\psi^{x}$ 's and $\beta_{X_{j}}$ 's. Hence, by Theorem 2.2 of ELP99, $\mathfrak{A} \cong \mathfrak{B}$.

If $X_{\square}=\emptyset$ the result is due to Elliott's classification result [Ell76], and if $X_{\square}=$ $\{m\}$ the theorem follows easily by making modifications to the above proof.

Remark 3.8. Let $\mathfrak{A}$ and $\mathfrak{B}$ be graph $C^{*}$-algebras that are $C^{*}$-algebras over $X$, so that $\mathfrak{A}\left(X_{i}\right)$ and $\mathfrak{B}\left(X_{i}\right)$ are tight $C^{*}$-algebras over $X_{i}$, for $i=1,2, \ldots, n$. Assume that

$0 \rightarrow \mathfrak{A}\left(X_{i}\right) / \mathfrak{A}\left(X_{i} \backslash\left\{x_{i}\right\}\right) \rightarrow \mathfrak{A}\left(X_{i} \cup\{m\}\right) / \mathfrak{A}\left(X_{i} \backslash\left\{x_{i}\right\}\right) \rightarrow \mathfrak{A}\left(X_{i} \cup\{m\}\right) / \mathfrak{A}\left(X_{i}\right) \rightarrow 0$ is essential whenever $\mathfrak{A}\left(X_{i}\right)$ is $\mathcal{O}_{\infty}$-absorbing, where $x_{i}$ is the greatest element of $X_{i}$. Assume that there exists an isomorphism $\alpha: \mathrm{FK}_{X}^{+}(\mathfrak{A}) \rightarrow \mathrm{FK}_{X}^{+}(\mathfrak{B})$. Assume moreover, that $\mathfrak{A}(\{m\})$ is an $A F$ algebra and that the set of $x \in X$ for which $\mathfrak{A}(\{x\})$ is an $A F$ algebra is hereditary. Then $\mathfrak{A} \otimes \mathbb{K} \cong \mathfrak{B} \otimes \mathbb{K}$. This follows from the proof above.

The above extensions are essential, e.g., if $\mathfrak{A}\left(\left\{x_{i}\right\}\right)$ is the least ideal of $\mathfrak{A}\left(\left\{x_{i}, m\right\}\right)$, for all $i=1,2, \ldots, n$, and the remark applies to the cases 3

(a) 4.E.1, where we view the algebra $\mathfrak{A}$ that is tight over the space 4 .E as a $C^{*}$-algebra over $a \leftarrow b \rightarrow c$ as indicated by the assignment $b \rightarrow a \leftarrow b \rightarrow c$.

(b) 4.1E.1 and 4.1E.3, where we view the algebra $\mathfrak{A}$ that is tight over the space $4.1 \mathrm{E}$ as a $C^{*}$-algebra over $a \leftarrow b \rightarrow c$ as indicated by the assignment

(c) $4.3 \mathrm{E} .1$, where we view the algebra $\mathfrak{A}$ that is tight over the space $4.3 \mathrm{E}$ as a $C^{*}$-algebra over $a \leftarrow b \rightarrow c$ as indicated by the assignment

The following proposition follows from the results in [ET10.

Proposition 3.9. Let $\mathfrak{A}$ be a graph $C^{*}$-algebra with exactly one nontrivial ideal $\mathfrak{I}$. If $\mathfrak{A}$ is not an AF algebra, then $0 \rightarrow \mathfrak{I} \otimes \mathbb{K} \rightarrow \mathfrak{A} \otimes \mathbb{K} \rightarrow \mathfrak{A} / \mathfrak{I} \otimes \mathbb{K} \rightarrow 0$ is a full extension.

Using the UCT for accordion spaces (see $\mathrm{MNa}$ and $\mathrm{BK}$ ) and for many other four-point spaces under the added assumption of real rank zero as described in ARR12, the cases 3.6.2, 3.6.3, 4.38.8, 4.38.9, 4.38.B, can be classified using the following theorem.

Theorem 3.10. Let $\mathfrak{A}$ and $\mathfrak{B}$ be graph $C^{*}$-algebras that are tight $C^{*}$-algebras over $X$, with $X_{i}$ being a singleton, for each $i=1,2, \ldots, n$. Suppose there exists an isomorphism $\alpha: \mathrm{FK}_{X}^{+}(\mathfrak{A}) \rightarrow \mathrm{FK}_{X}^{+}(\mathfrak{B})$ which lifts to an invertible element in $K K(X ; \mathfrak{A}, \mathfrak{B})$. Then $\mathfrak{A} \otimes \mathbb{K} \cong \mathfrak{B} \otimes \mathbb{K}$.

\footnotetext{
${ }^{3}$ Here we specify how we view the algebras as algebras over $a \leftarrow b \rightarrow c$ by providing a continuous map from the primitive ideal space to $\{a, b, c\}$
} 
Proof. If $\mathfrak{A}(\{m\})$ is an $A F$ algebra, the result follows from Theorem 3.7. Suppose $\mathfrak{A}(\{m\})$ is an $\mathcal{O}_{\infty}$-absorbing simple $C^{*}$-algebra and that $\mathfrak{A}$ and $\mathfrak{B}$ are stable $C^{*}$-algebras. Then by Lemma 3.5 and Proposition 3.9, $\bar{\pi}_{i} \circ \eta_{\mathfrak{e}} \mathfrak{A}: \mathfrak{A}(\{m\}) \rightarrow \mathcal{Q}\left(\mathfrak{A}\left(X_{i}\right)\right)$ and $\bar{\pi}_{i} \circ \eta_{\mathfrak{e}^{\mathfrak{B}}}: \mathfrak{B}(\{m\}) \rightarrow \mathcal{Q}\left(\mathfrak{B}\left(X_{i}\right)\right)$ are full extensions, for all $i=1,2, \ldots, n$. Hence, by Lemma 3.6, $\eta_{\mathfrak{e}^{\mathfrak{A}}}$ and $\eta_{\mathfrak{e}^{\mathfrak{B}}}$ are full extensions. The theorem now follows from the results of [ERRa].

\subsection{Primitive ideal space with $n$ minimal elements.}

Assumption 3.11. For this subsection, let $n>1$ be a fixed integer, and let $X_{i}=\mathrm{X}_{l_{i}}$ for $i=1,2, \ldots, n$, where $l_{1}, l_{2}, \ldots, l_{n}$ are fixed positive integers. Let, moreover,

$$
X=\{M\} \sqcup X_{1} \sqcup X_{2} \sqcup \cdots \sqcup X_{n}
$$

and define a partial order on $X$ as follows. The element $M$ is the greatest element of $X$, and for each $i=1,2, \ldots, n$, if $x, y \in X_{i}$ then $x \leq y$ in $X$ if and only if $x \leq y$ in $X_{i}$. No other relations are between the elements of $X$.

Lemma 3.12. Let $\mathfrak{A}$ be a tight $C^{*}$-algebra over $X$ and let $Y \in \mathbb{O}\left(X_{\square} \backslash\{M\}\right)$ be given. Consider the extensions

$$
\mathfrak{e}: 0 \rightarrow \mathfrak{A}(\{M\}) \rightarrow \mathfrak{A} \rightarrow \mathfrak{A}(X \backslash\{M\}) \rightarrow 0
$$

and

$$
\iota_{\mathfrak{A}, Y} \cdot \mathfrak{e}: 0 \rightarrow \mathfrak{A}(\{M\}) \rightarrow \mathfrak{A}(Y \cup\{M\}) \rightarrow \mathfrak{A}(Y) \rightarrow 0
$$

where $\iota_{\mathfrak{A}, Y}: \mathfrak{A}(Y) \rightarrow \mathfrak{A}(X \backslash\{M\})$ is the usual embedding. Then $\eta_{\iota_{\mathfrak{A}, Y} \cdot \mathfrak{e}}=\eta_{\mathfrak{e}} \circ \iota_{\mathfrak{A}, Y}$.

Proof. Note that the diagram

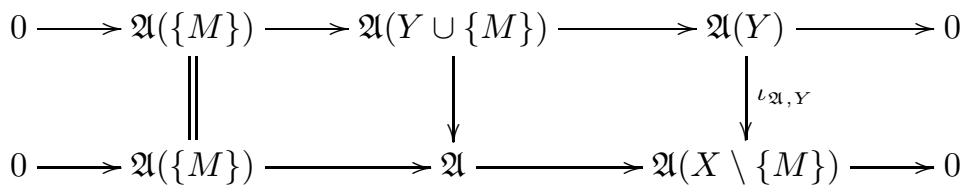

commutes. Hence, by Theorem 2.2 of [ELP99], $\eta_{\iota_{\mathfrak{A}, Y} \cdot \mathfrak{e}}=\eta_{\mathfrak{e}} \circ \iota_{\mathfrak{A}, Y}$.

Lemma 3.13. Suppose the following diagram of $C^{*}$-algebras with short exact rows is commutative

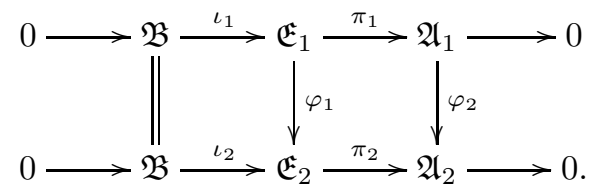

(1) If $\varphi_{2}\left(\mathfrak{A}_{1}\right)$ is a hereditary sub-C $C^{*}$-algebra of $\mathfrak{A}_{2}$, then $\varphi_{1}\left(\mathfrak{E}_{1}\right)$ is a hereditary sub-C $C^{*}$-algebra of $\mathfrak{E}_{2}$.

(2) If $\varphi_{2}\left(\mathfrak{A}_{1}\right)$ is full in $\mathfrak{A}_{2}$, then $\varphi_{1}\left(\mathfrak{E}_{1}\right)$ is full in $\mathfrak{E}_{2}$.

Proof. We first prove (1). Let $x \in \mathfrak{E}_{1}$ and $y \in \mathfrak{E}_{2}$ such that $0 \leq y \leq \varphi_{1}(x)$. Since $\varphi_{2}\left(\mathfrak{A}_{1}\right)$ is a hereditary sub- $C^{*}$-algebra of $\mathfrak{A}_{2}$, we have that there exists $z \in \varphi_{1}\left(\mathfrak{E}_{1}\right)$ such that $\pi_{2}(y)=\pi_{2}(z)$. Thus, $y-z \in \mathfrak{B}$. Since the map on the ideals is the identity, we have that $y-z \in \varphi_{1}\left(\mathfrak{E}_{1}\right)$. Hence, $y \in \varphi_{1}\left(\mathfrak{E}_{1}\right)$. Therefore, $\varphi_{1}\left(\mathfrak{E}_{1}\right)$ is a hereditary sub- $C^{*}$-algebra of $\mathfrak{E}_{2}$.

We now prove (2). Let $x \in \mathfrak{E}_{2}$. Since $\varphi_{2}\left(\mathfrak{A}_{1}\right)$ is full in $\mathfrak{A}_{2}$, there exists $y$ in the ideal of $\mathfrak{E}_{2}$ generated by $\varphi_{1}\left(\mathfrak{E}_{1}\right)$ such that $x-y \in \mathfrak{B}$. Since the map on the ideals is 
the identity, we have that $y-z \in \varphi_{1}\left(\mathfrak{E}_{1}\right)$. Hence, $x$ is in the ideal of $\mathfrak{E}_{2}$ generated by $\varphi_{1}\left(\mathfrak{E}_{1}\right)$.

Lemma 3.14. Let $\mathfrak{e}: 0 \rightarrow \mathfrak{I} \rightarrow \mathfrak{A} \rightarrow \bigoplus_{k=1}^{n} \mathfrak{A}_{k} \rightarrow 0$ be an extension and let $\iota_{k}: \mathfrak{A}_{k} \rightarrow \bigoplus_{k=1}^{n} \mathfrak{A}_{k}$ be the inclusion. Suppose $\eta_{\mathfrak{e}} \circ \iota_{k}$ is full for each $k$. Then $\eta_{\mathfrak{e}}$ is full.

Proof. Let $\left(a_{1}, a_{2}, \ldots, a_{n}\right)$ be a nonzero positive element in $\bigoplus_{k=1}^{n} \mathfrak{A}_{k}$. Without loss of generality, we may assume that $a_{1} \neq 0$. Note that ideal in $\mathcal{Q}(\mathfrak{I})$ generated by $\eta_{\mathfrak{e}}\left(a_{1}, \ldots, a_{n}\right)$ contains the ideal in $\mathcal{Q}(\mathfrak{I})$ generated by $\eta_{\mathfrak{e}} \circ \iota_{1}\left(a_{1}\right)$. Since $\eta_{\mathfrak{e}} \circ \iota_{k}$ is full, we have that the ideal in $\mathcal{Q}(\mathfrak{I})$ generated by $\eta_{\mathfrak{e}} \circ \iota_{1}\left(a_{1}\right)$ is $\mathcal{Q}(\mathfrak{I})$. Thus, the ideal in $\mathcal{Q}(\mathfrak{I})$ generated by $\eta_{\mathfrak{e}}\left(a_{1}, \ldots, a_{n}\right)$ is $\mathcal{Q}(\mathfrak{I})$.

The following result applies to the cases 3.3.1, 3.3.5, 4.F.6, 4.F.8, 4.F.E, 4.A.2, 4.A.6, 4.A.E.

Theorem 3.15. Let $\mathfrak{A}$ and $\mathfrak{B}$ be graph $C^{*}$-algebras that are tight $C^{*}$-algebras over $X$ such that each of $\mathfrak{A}\left(X_{i}\right), \mathfrak{B}\left(X_{i}\right)$ are either $A F$ algebras or $\mathcal{O}_{\infty}$-absorbing. Suppose there exists an isomorphism $\alpha: \mathrm{FK}_{X}^{+}(\mathfrak{A}) \rightarrow \mathrm{FK}_{X}^{+}(\mathfrak{B})$ and $\mathfrak{A}(\{M\})$ is an $A F$ algebra. Then $\mathfrak{A} \otimes \mathbb{K} \cong \mathfrak{B} \otimes \mathbb{K}$.

Proof. We may assume that $\mathfrak{A}$ and $\mathfrak{B}$ are stable $C^{*}$-algebras. Note that for each $x \in X, \mathfrak{A}(\{x\})$ is an $A F$ algebra if and only if $\mathfrak{B}(\{x\})$ is an $A F$ algebra, and $\mathfrak{A}(\{x\})$ is $\mathcal{O}_{\infty}$-absorbing if and only if $\mathfrak{B}(\{x\})$ is $\mathcal{O}_{\infty}$-absorbing (since there exists a positive isomorphism from $K_{0}\left(\mathfrak{A}(\{x\})\right.$ to $K_{0}(\mathfrak{B}(\{x\}))$. Specifically, $\mathfrak{B}(\{M\})$ is an $A F$ algebra. First we assume that $X_{\square} \neq \emptyset$ and $X_{\square} \backslash\{M\} \neq \emptyset$.

Note that $\mathfrak{A}\left(X_{\square}\right)$ and $\mathfrak{B}\left(X_{\square}\right)$ are $A F$ algebras. Since $\alpha_{X_{\square}}: K_{0}\left(\mathfrak{A}\left(X_{\square}\right)\right) \rightarrow$ $K_{0}\left(\mathfrak{B}\left(X_{\square}\right)\right)$ is a positive isomorphism, there exists an isomorphism $\beta: \mathfrak{A}\left(X_{\square}\right) \rightarrow$ $\mathfrak{B}\left(X_{\square}\right)$ such that $K_{0}(\beta)=\alpha_{X_{\square}}$ (by Elliott's classification result [Ell76]). Since $\mathfrak{A}\left(X_{\square}\right)$ and $\mathfrak{B}\left(X_{\square}\right)$ are $A F$ algebras and $\beta$ is an $X_{\square}$-equivariant isomorphism, we have that $K_{0}\left(\beta_{Y}\right)=\alpha_{Y}$ for all $Y \in \mathbb{L} \mathbb{C}(X)$ such that $Y \subseteq X_{\square}$. In particular, $K_{0}\left(\beta_{\{x\}}\right)=\alpha_{\{x\}}$ for all $x \in X_{\square}$.

Let

and

$$
\mathfrak{e}_{\mathfrak{A}}: 0 \rightarrow \mathfrak{A}(\{M\}) \rightarrow \mathfrak{A} \rightarrow \mathfrak{A}(X \backslash\{M\}) \rightarrow 0,
$$

$$
\mathfrak{e}_{\mathfrak{B}}: 0 \rightarrow \mathfrak{B}(\{M\}) \rightarrow \mathfrak{B} \rightarrow \mathfrak{B}(X \backslash\{M\}) \rightarrow 0 .
$$

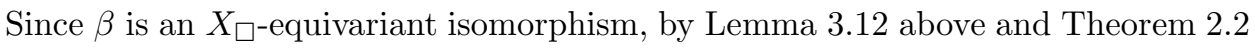
of [ELP99], for $Y \in \mathbb{O}\left(X_{\square} \backslash\{M\}\right)$

$$
\bar{\beta}_{\{M\}} \circ \eta_{\mathfrak{e}_{\mathfrak{A}}} \circ \iota_{\mathfrak{A}, Y}=\eta_{\mathfrak{e}_{B}} \circ \iota_{\mathfrak{B}, Y} \circ \beta_{Y}
$$

for all $Y \in \mathbb{O}\left(X_{\square} \backslash\{M\}\right)$, where $\iota_{\mathfrak{A}, Y}: \mathfrak{A}(Y) \rightarrow \mathfrak{A}(X \backslash\{M\})$ and $\iota_{\mathfrak{B}, Y}: \mathfrak{B}(Y) \rightarrow$ $\mathfrak{B}(X \backslash\{M\})$ are the canonical embeddings.

Since $\alpha$ induces an isomorphism reaching from $\mathrm{FK}_{X \mathbf{\square} \cup M\}}^{+}(\mathfrak{A}(X \mathbb{\square} \cup\{M\}))$ to $\mathrm{FK}_{X \backsim\{M\}}^{+}\left(\mathfrak{B}\left(X_{\mathbf{\square}} \cup\{M\}\right)\right)$, by Lemma3.12, Theorem 2.3 of [ERR09, Theorem 4.14 of [MNa, Kirchberg [Kir00, and Theorem 3.3 of [ERRa]), there exists an $X_{\mathbf{m}}$ equivariant isomorphism $\psi: \mathfrak{A}\left(X_{\mathbf{\square}}\right) \rightarrow \mathfrak{B}\left(X_{\mathbf{m}}\right)$ such that $K_{*}(\psi)=\alpha_{X}$ and

$$
\left[\bar{\beta}_{\{M\}} \circ \eta_{\mathfrak{e}_{\mathfrak{A}}} \circ \iota_{\mathfrak{A}, X \mathbf{\square}}\right]=\left[\eta_{\mathfrak{e}_{\mathfrak{B}}} \circ \iota_{\mathfrak{B}, X \mathbf{\square}} \circ \psi\right]
$$

in $K K^{1}(\mathfrak{A}(X \mathbf{\square}), \mathfrak{B}(\{M\}))$. By Corollary 5.6 of ERRa, $\eta_{\mathfrak{e}_{\mathfrak{A}}} \circ \iota_{\mathfrak{A}, X_{i}}$ and $\eta_{\mathfrak{e}_{\mathfrak{B}}} \circ$ $\iota_{\mathfrak{B}, X_{i}}$ are full extensions for each $i=1,2, \ldots, n$ with $X_{i}$ being $\mathcal{O}_{\infty}$-absorbing 
(i.e., $X_{i} \subseteq X_{\mathbf{\square}}$ ). Thus, $\eta_{\mathfrak{e}_{\mathfrak{A}}} \circ \iota_{\mathfrak{A}, X_{\mathbf{\square}}}$ and $\eta_{\mathfrak{e}_{\mathfrak{B}}} \circ \iota_{\mathfrak{B}, X_{\mathbf{\square}}}$ are full extensions since $\mathfrak{A}\left(X_{\mathbf{\square}}\right)=\bigoplus_{i \in\{1,2, \ldots, n\}, X_{i} \subseteq X_{\mathbf{\square}}} \mathfrak{A}\left(X_{i}\right)$ and $\mathfrak{B}\left(X_{\mathbf{\square}}\right)=\bigoplus_{i \in\{1,2, \ldots, n\}, X_{i} \subseteq X_{\mathbf{\square}}} \mathfrak{B}\left(X_{i}\right)$. Hence, $\bar{\beta}_{\{M\}} \circ \eta_{\mathfrak{e}_{\mathfrak{A}}} \circ \iota_{\mathfrak{A}, X \boldsymbol{\square}}$ and $\eta_{\mathfrak{e}_{\mathfrak{B}}} \circ \iota_{\mathfrak{B}, X \mathbf{\square}} \circ \psi$ are full extensions.

Let $\pi_{\mathfrak{A}, X_{\square} \backslash\{M\}}: \mathfrak{A}(X \backslash\{M\}) \rightarrow \mathfrak{A}\left(X_{\square} \backslash\{M\}\right), \pi_{\mathfrak{A}, X_{\mathbf{\square}}}: \mathfrak{A}(X \backslash\{M\}) \rightarrow \mathfrak{A}\left(X_{\mathbf{\square}}\right)$, $\pi_{\mathfrak{B}, X_{\square} \backslash\{M\}}: \mathfrak{B}(X \backslash\{M\}) \rightarrow \mathfrak{B}\left(X_{\square} \backslash\{M\}\right), \pi_{\mathfrak{B}, X}: \mathfrak{B}(X \backslash\{M\}) \rightarrow \mathfrak{B}\left(X_{\mathbf{\square}}\right)$ be the canonical projections. Note that the range of $\eta_{\mathfrak{e}_{\mathfrak{A}}} \circ \iota_{\mathfrak{A}, X_{\square} \backslash\{M\}}$ and the range of $\eta_{\mathfrak{e}_{\mathfrak{A}}} \circ \iota_{\mathfrak{A}, X_{\mathbf{\square}}}$ are orthogonal and the range of $\eta_{\mathfrak{e}_{\mathfrak{B}}} \circ \iota_{\mathfrak{B}, X_{\square} \backslash\{M\}}$ and the range of $\eta_{\mathfrak{e}_{\mathfrak{B}}} \circ \iota_{\mathfrak{B}, X \boldsymbol{\square}}$ are orthogonal. Moreover,

$$
\begin{aligned}
\eta_{\mathfrak{e}_{\mathfrak{A}}} & =\eta_{\mathfrak{e}_{\mathfrak{A}}} \circ \iota_{\mathfrak{A}, X_{\square} \backslash\{M\}} \circ \pi_{\mathfrak{A}, X_{\square} \backslash\{M\}}+\eta_{\mathfrak{e}_{\mathfrak{A}}} \circ \iota_{\mathfrak{A}, X_{\mathbf{\square}}} \circ \pi_{\mathfrak{A}, X} \\
\eta_{\mathfrak{e}_{\mathfrak{B}}} & =\eta_{\mathfrak{e}_{\mathfrak{B}}} \circ \iota_{\mathfrak{B}, X_{\square} \backslash\{M\}} \circ \pi_{\mathfrak{B}, X_{\square} \backslash\{M\}}+\eta_{\mathfrak{e}_{\mathfrak{B}}} \circ \iota_{\mathfrak{B}, X_{\square}} \circ \pi_{\mathfrak{B}, X} .
\end{aligned}
$$

We claim that there exist full hereditary sub- $C^{*}$-algebras $\mathcal{E}_{1}$ and $\mathcal{E}_{2}$ of $\mathfrak{A}$ and $\mathfrak{B}$, respectively, such that $\mathcal{E}_{1} \cong \mathcal{E}_{2}$. Then by Theorem 2.8 of [Bro77], $\mathfrak{A} \otimes \mathbb{K} \cong \mathfrak{B} \otimes \mathbb{K}$.

Choose full projections $p_{1}, q_{1} \in \mathfrak{A}\left(X_{\mathbf{\square}}\right)$ and $p_{2}, q_{2} \in \mathfrak{A}\left(X_{\square} \backslash\{M\}\right)$ such that $p_{1}+p_{2}$ is orthogonal to $q_{1}+q_{2}$ in $\mathfrak{A}(X \backslash\{M\})$ (to do this, we use stability, and that graph algebras with finitely many ideals satisfies Condition $(K)$ and hence are of real rank zero). Therefore, $\eta_{\mathfrak{e}_{\mathfrak{A}}}\left(p_{1}+p_{2}\right) \neq 1_{\mathcal{Q}(\mathfrak{A}(\{M\}))}$ since $\eta_{\mathfrak{e}_{\mathfrak{A}}}\left(p_{1}+p_{2}\right)$ is orthogonal to $\eta_{\mathfrak{e}_{\mathfrak{A}}}\left(q_{1}+q_{2}\right)$. Set $e_{1}=\psi\left(p_{1}\right), e_{2}=\beta_{X_{\square} \backslash\{M\}}\left(p_{2}\right), f_{1}=\psi\left(q_{1}\right)$, and $f_{2}=\beta_{X_{\square} \backslash\{M\}}\left(q_{2}\right)$. Then $e_{1}+e_{2}$ and $f_{1}+f_{2}$ are nonzero orthogonal projections. So, $\eta_{\mathfrak{e}_{\mathfrak{B}}}\left(e_{1}+e_{2}\right) \neq 1_{\mathcal{Q}(\mathfrak{B}(\{M\}))}$.

Set $e=\bar{\beta}_{\{M\}} \circ \eta_{\mathfrak{e}_{\mathfrak{A}}} \circ \iota_{\mathfrak{A}, X_{\square} \backslash\{M\}}\left(p_{2}\right)=\eta_{\mathfrak{e}_{\mathfrak{B}}} \circ \iota_{\mathfrak{B}, X_{\square} \backslash\{M\}} \circ \beta_{X_{\square} \backslash\{M\}}\left(p_{2}\right)$ and set $f=\left(1_{\mathcal{Q}(\mathfrak{B}(\{M\}))}-e\right)$. Let $j_{\mathbf{\square}}: p_{1} \mathfrak{A}\left(X_{\mathbf{\square}}\right) p_{1} \rightarrow \mathfrak{A}\left(X_{\mathbf{\square}}\right)$ and $j_{\square}: p_{2} \mathfrak{A}\left(X_{\square} \backslash\{M\}\right) p_{2} \rightarrow$ $\mathfrak{A}\left(X_{\square} \backslash\{M\}\right)$ be the usual embeddings. Note that

$$
e \bar{\beta}_{\{M\}} \circ \eta_{\mathfrak{e}_{\mathfrak{A}}} \circ \iota_{\mathfrak{A}, \boldsymbol{\square}} \circ j \mathbf{\square}(x)=\bar{\beta}_{\{M\}} \circ \eta_{\mathfrak{e}_{\mathfrak{A}}} \circ \iota_{\mathfrak{A}, \boldsymbol{\square}} \circ j \mathbf{\square}(x) e=0
$$

and

$$
\begin{gathered}
e\left(\eta_{\mathfrak{e}_{\mathfrak{B}}} \circ \iota_{\mathfrak{B}, X \boldsymbol{\square}} \circ \psi \circ j \mathbf{\square}(x)\right)= \\
\left(\eta_{\mathfrak{e}_{\mathfrak{B}}} \circ \iota_{\mathfrak{B}, X_{\square} \backslash\{M\}} \circ \beta_{X_{\square} \backslash\{M\}}\left(p_{2}\right)\right) \cdot\left(\eta_{\mathfrak{e}_{\mathfrak{B}}} \circ \iota_{\mathfrak{B}, X \boldsymbol{\square}} \circ \psi \circ j_{\mathbf{\square}}(x)\right)=0
\end{gathered}
$$

as well as

$$
\begin{gathered}
\left(\eta_{\mathfrak{e}_{\mathfrak{B}}} \circ \iota_{\mathfrak{B}, X \boldsymbol{\square}} \circ \psi \circ j \boldsymbol{\square}(x)\right) e= \\
\left(\eta_{\mathfrak{e}_{\mathfrak{B}}} \circ \iota_{\mathfrak{B}, X \boldsymbol{\square}} \circ \psi \circ j \mathbf{\square}(x)\right) \cdot\left(\eta_{\mathfrak{e}_{\mathfrak{B}}} \circ \iota_{\mathfrak{B}, X_{\square} \backslash\{M\}} \circ \beta_{X_{\square} \backslash\{M\}}\left(p_{2}\right)\right)=0
\end{gathered}
$$

for all $x \in p_{1} \mathfrak{A}\left(X_{\boldsymbol{\square}}\right) p_{1}$. Hence, we have injective homomorphisms $\bar{\beta}_{\{M\}} \circ \eta_{\mathfrak{e}_{\mathfrak{A}}} \circ \iota_{\mathfrak{A}, \boldsymbol{\bullet}}$

$j \mathbf{\square}$ and $\eta_{\mathfrak{e}_{\mathfrak{B}}} \circ \iota_{\mathfrak{B}, X \boldsymbol{\square}} \circ \psi \circ j \boldsymbol{\square}$ from $p_{1} \mathfrak{A}\left(X_{\mathbf{\square}}\right) p_{1}$ to $f \mathcal{Q}(\mathfrak{B}(\{M\})) f$.

Since $\mathfrak{B}(\{M\})$ is an $A F$ algebra, by Corollary 2.11 of Zha91 $f$ lifts to a projection $f^{\prime}$ in $\mathcal{M}(\mathfrak{B}(\{M\}))$. Note that there exists an isomorphism $\gamma$ from $f^{\prime} \mathcal{M}(\mathfrak{B}(\{M\})) f^{\prime}$ to $\mathcal{M}\left(f^{\prime} \mathfrak{B}(\{M\}) f^{\prime}\right)$ which is the identity on $f^{\prime} \mathfrak{B}(\{M\}) f^{\prime}$ (see II.7.3.14, pp. 147 of [BB] $)$. Thus, we have an isomorphism $\bar{\gamma}$ from $f \mathcal{Q}(\mathfrak{B}(\{M\})) f$ to $\mathcal{Q}\left(f^{\prime} \mathfrak{B}(\{M\}) f^{\prime}\right)$ such that the diagram

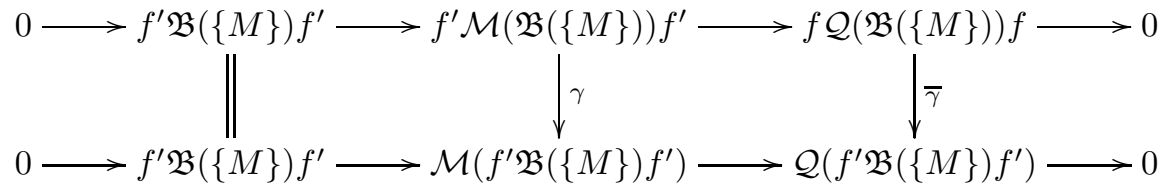

is commutative. By Corollary 5.6 of ERRa, $\eta_{\mathfrak{e}_{\mathfrak{A}}} \circ \iota_{\mathfrak{A}, X_{i}}$ and $\eta_{\mathfrak{e}_{\mathfrak{B}}} \circ \iota_{\mathfrak{B}, X_{i}}$ are full extensions for each $i=1,2, \ldots, n$ with $X_{i}$ being $\mathcal{O}_{\infty}$-absorbing (i.e., $X_{i} \subseteq$ 
$X_{\mathbf{\square}}$. Thus, by Lemma 3.14, $\eta_{\mathfrak{e}_{\mathfrak{A}}} \circ \iota_{\mathfrak{A}, X \mathbb{\square}}$ and $\eta_{\mathfrak{e}_{\mathfrak{B}}} \circ \iota_{\mathfrak{B}, X \mathbf{\Xi}}$ are full extensions since $\mathfrak{A}\left(X_{\mathbf{m}}\right)=\bigoplus_{i \in\{1,2, \ldots, n\}, X_{i} \subseteq X_{\mathbf{m}}} \mathfrak{A}\left(X_{i}\right)$ and $\mathfrak{B}\left(X_{\mathbf{m}}\right)=\bigoplus_{i \in\{1,2, \ldots, n\}, X_{i} \subseteq X \mathbf{\square}} \mathfrak{B}\left(X_{i}\right)$. Hence, $\bar{\beta}_{\{M\}} \circ \eta_{\mathfrak{e}_{\mathfrak{A}}} \circ \iota_{\mathfrak{A}, X \mathbb{\square}}$ and $\eta_{\mathfrak{e}_{\mathfrak{B}}} \circ \iota_{\mathfrak{B}, X \mathbf{\square}} \circ \psi$ are full extensions. Thus, $\bar{\beta}_{\{M\}} \circ \eta_{\mathfrak{e}_{\mathfrak{A} A}} \circ$ $\iota_{\mathfrak{A}, X \mathbb{\square}}\left(p_{1}\right)$ is a norm-full projection in $\mathcal{Q}(\mathfrak{B}(\{M\}))$. Since $\bar{\beta}_{\{M\}} \circ \eta_{\mathfrak{e}_{\mathfrak{A}}} \circ \iota_{\mathfrak{A}, X \mathbb{\square}}\left(p_{1}\right) \leq f$, we have that $f$ is a norm-full projection in $\mathcal{Q}(\mathfrak{B}(\{M\}))$. By Lemma 3.3 of [ERRb, we have that $f^{\prime}$ is a norm-full projection in $\mathcal{M}(\mathfrak{B}(\{M\}))$ since $\mathfrak{B}(\{M\})$ has an approximate identity consisting of projections. Since $\mathfrak{B}(\{M\})$ is an $A F$ algebra, by Lemma 3.10 of ERR09, $\mathfrak{B}(\{M\})$ has the corona factorization property. Thus, $f^{\prime}$

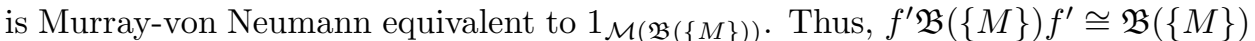
which implies that $f^{\prime} \mathfrak{B}(\{M\}) f^{\prime}$ is a stable $C^{*}$-algebra since $\mathfrak{B}(\{M\})$ is a stable $C^{*}$-algebra.

Let $\iota$ be the embedding of $f^{\prime} \mathfrak{B}(\{M\}) f^{\prime}$ into $\mathfrak{B}(\{M\}), \widetilde{\iota}$ be the embedding of $f^{\prime} \mathcal{M}(\mathfrak{B}(\{M\})) f^{\prime}$ into $\mathcal{M}(\mathfrak{B}(\{M\}))$, and $\bar{\iota}$ be the embedding of $f \mathcal{Q}(\mathfrak{B}(\{M\})) f$ into $\mathcal{Q}(\mathfrak{B}(\{M\}))$. Note that the following diagram

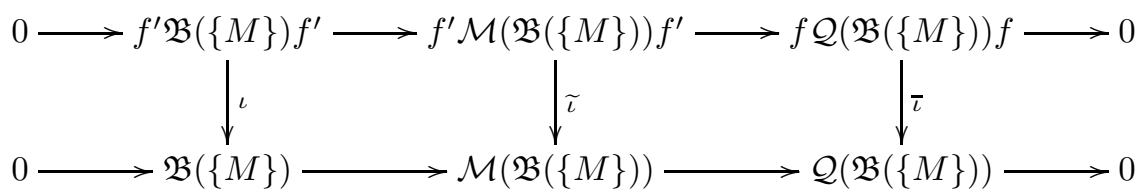

is commutative. Note that the range of $\eta_{\mathfrak{e}_{\mathfrak{B}}} \circ \iota_{\mathfrak{B}, X \boldsymbol{\mathbf { m }}} \circ \psi \circ j \mathbf{m}$ and the range of $\bar{\beta}_{\{M\}} \circ \eta_{\mathfrak{e}_{\mathfrak{A}}} \circ \iota_{\mathfrak{A}, X \boldsymbol{\square}} \circ j \mathbf{\square}$ are contained in $f \mathcal{Q}(\mathfrak{B}(\{M\})) f$. Let $\mathfrak{e}_{1}$ be the extension defined by $\bar{\gamma} \circ \bar{\iota}^{-1} \circ \bar{\beta}_{\{M\}} \circ \eta_{\mathfrak{e}_{\mathfrak{A}}} \circ \iota_{\mathfrak{A}, X \boldsymbol{\square}} \circ j \mathbf{\square}$ and let $\mathfrak{e}_{2}$ be the extension defined by $\bar{\gamma} \circ \bar{\iota}^{-1} \circ \eta_{\mathfrak{e}_{\mathfrak{B}}} \circ \iota_{\mathfrak{B}, X \boldsymbol{\square}} \circ \psi \circ j \mathbf{m}$. Then $\bar{\iota} \circ \bar{\gamma}^{-1} \circ \eta_{\mathfrak{e}_{1}}=\bar{\beta}_{\{M\}} \circ \eta_{\mathfrak{e}_{\mathfrak{A}}} \circ \iota_{\mathfrak{A}, X \mathbf{\square}} \circ j \mathbf{\square}$ and $\bar{\iota} \circ \bar{\gamma}^{-1} \circ \eta_{\mathfrak{e}_{2}}=\eta_{\mathfrak{e}_{\mathfrak{B}}} \circ \iota_{\mathfrak{B}, X \mathbb{\square}} \circ \psi \circ j$

Since $\eta_{\mathfrak{e}_{\mathfrak{A}}}\left(p_{1}+p_{2}\right) \neq 1_{\mathcal{Q}(\mathfrak{A}(\{M\}))}$ and $\eta_{\mathfrak{e}_{\mathfrak{B}}}\left(e_{1}+e_{2}\right) \neq 1_{\mathcal{Q}(\mathfrak{B}(\{M\}))}$ and since $\bar{\beta}_{\{M\}}$ and $\psi$ are isomorphisms, we have that $\bar{\beta}_{\{M\}} \circ \eta_{\mathfrak{e}_{\mathfrak{A}}} \circ \iota_{\mathfrak{A}, X \mathbf{\square}} \circ j_{\mathbf{m}}\left(p_{1}\right) \neq f$ and $\eta_{\mathfrak{e}_{\mathfrak{B}}} \circ$ $\iota_{\mathfrak{B}, X \boldsymbol{\square}} \circ \psi \circ j_{\mathbf{\square}}\left(p_{1}\right) \neq f$. Thus, $\eta_{\mathfrak{e}_{1}}\left(p_{1}\right)$ and $\eta_{\mathfrak{e}_{2}}\left(p_{1}\right)$ are not equal to $1_{\mathcal{Q}\left(f^{\prime} \mathfrak{B}(\{M\}) f^{\prime}\right)}$. Therefore, $\mathfrak{e}_{1}$ and $\mathfrak{e}_{2}$ are non-unital full extensions. Since

$$
\left[\bar{\beta}_{\{M\}} \circ \eta_{\mathfrak{e}_{\mathfrak{A}}} \circ \iota_{\mathfrak{A}, X \mathbf{\square}}\right]=\left[\eta_{\mathfrak{e}_{\mathfrak{B}}} \circ \iota_{\mathfrak{B}, X \mathbf{\square}} \circ \psi\right]
$$

in $K K^{1}(\mathfrak{A}(X \mathbf{\square}), \mathfrak{B}(\{M\}))$, since $\iota$ induces an element in $K K\left(f^{\prime} \mathfrak{B}(\{M\}) f^{\prime}, \mathfrak{B}(\{M\})\right)$ which is invertible, and since $\bar{\gamma}$ is an isomorphism, we have that $\left[\eta_{\mathfrak{e}_{1}}\right]=\left[\eta_{\mathfrak{e}_{2}}\right]$ in $K K^{1}\left(p_{1} \mathfrak{A}(X \mathbf{\square}) p_{1}, f^{\prime} \mathfrak{B}(\{M\}) f^{\prime}\right)$. Since $f^{\prime} \mathfrak{B}(\{M\}) f^{\prime} \cong \mathfrak{B}(\{M\})$, we have that $f^{\prime} \mathfrak{B}(\{M\}) f^{\prime}$ has the corona factorization property. Thus, there exists a unitary $u^{\prime}$ in $\mathcal{M}\left(f^{\prime} \mathfrak{B}(\{M\}) f^{\prime}\right)$ such that

$$
\operatorname{Ad}\left(\overline{u^{\prime}}\right) \circ \eta_{\mathfrak{e}_{1}}=\eta_{\mathfrak{e}_{2}}
$$

where $\overline{u^{\prime}}$ is the image of $u^{\prime}$ in $\mathcal{Q}\left(f^{\prime} \mathfrak{B}(\{M\}) f^{\prime}\right)$. Let $u=\widetilde{\iota} \circ \gamma^{-1}\left(u^{\prime}\right)$. Then $u$ is a partial isometry in $\mathcal{M}(\mathfrak{B}(\{M\}))$ such that $u^{*} u=f^{\prime}=u u^{*}$ and

$$
\operatorname{Ad}(\bar{u}) \circ \bar{\beta}_{\{M\}} \circ \eta_{\mathfrak{e}_{\mathfrak{A}}} \circ \iota_{\mathfrak{A}, X \mathbf{\square}} \circ j \mathbf{\square}=\eta_{\mathfrak{e}_{\mathfrak{B}}} \circ \iota_{\mathfrak{B}, X \mathbf{\square}} \circ \psi \circ j
$$

where $\bar{u}$ is the image of $u$ in $\mathcal{Q}(\mathfrak{B}(\{M\}))$. Set $v=u+1_{\mathcal{M}(\mathfrak{B}(\{M\}))}-f^{\prime}$ and let $\bar{v}$ be the image of $v$ in $\mathcal{Q}(\mathfrak{B}(\{M\}))$. Note that $\bar{v}=\bar{u}+e$ and

$$
\begin{array}{r}
\operatorname{Ad}(\bar{v}) \circ \bar{\beta}_{\{M\}} \circ \eta_{\mathfrak{e}_{\mathfrak{A}}} \circ \iota_{\mathfrak{A}, X_{\square} \backslash\{M\}} \circ j_{\square}=\bar{\beta}_{\{M\}} \circ \eta_{\mathfrak{e}_{\mathfrak{A}}} \circ \iota_{\mathfrak{A}, X_{\square} \backslash\{M\}} \circ j_{\square} \\
\operatorname{Ad}(\bar{v}) \circ \bar{\beta}_{\{M\}} \circ \eta_{\mathfrak{e}_{\mathfrak{A}}} \circ \iota_{\mathfrak{A}, X \boldsymbol{\square}} \circ j_{\mathbf{\square}}=\eta_{\mathfrak{e}_{\mathfrak{B}}} \circ \iota_{\mathfrak{B}, X \boldsymbol{\square}} \circ \psi \circ j \mathbf{\square} .
\end{array}
$$


Let $a_{1} \in p_{1} \mathfrak{A}\left(X_{\square}\right) p_{1}$ and $a_{2} \in p_{2} \mathfrak{A}\left(X_{\square} \backslash\{M\}\right) p_{2}$. Then

$$
\begin{aligned}
& \bar{v}\left(\bar{\beta}_{\{M\}} \circ \eta_{\mathfrak{e}_{\mathfrak{A}}} \circ \iota_{\mathfrak{A}, X \square} \circ j_{\square}\left(a_{1}\right)+\bar{\beta}_{\{M\}} \circ \eta_{\mathfrak{e}_{\mathfrak{A}}} \circ \iota_{\mathfrak{A}, X_{\square} \backslash\{M\}} \circ j_{\square}\left(a_{2}\right)\right) \bar{v}^{*} \\
& =\eta_{\mathfrak{e}_{\mathfrak{B}}} \circ \iota_{\mathfrak{B}, X \llbracket} \circ \psi \circ j \mathbf{\square}\left(a_{1}\right)+\bar{\beta}_{\{M\}} \circ \eta_{\mathfrak{e}_{\mathfrak{A}}} \circ \iota_{\mathfrak{A}, X_{\square} \backslash\{M\}} \circ j_{\square}\left(a_{2}\right) \\
& =\eta_{\mathfrak{e}_{\mathfrak{B}}} \circ \iota_{\mathfrak{B}, X \mathbf{\square}} \circ \psi \circ j_{\mathbf{\square}}\left(a_{1}\right)+\eta_{\mathfrak{e}_{\mathfrak{B}}} \circ \iota_{\mathfrak{B}, X_{\square} \backslash\{M\}} \circ \beta_{X_{\square} \backslash\{M\}} \circ j_{\square}\left(a_{2}\right) \\
& =\eta_{\mathfrak{e}_{\mathfrak{B}}} \circ\left(\psi \circ j_{\square}\left(a_{1}\right)+\beta_{X_{\square} \backslash\{M\}} \circ j_{\square}\left(a_{2}\right)\right) \text {. }
\end{aligned}
$$

Hence,

(1) $\operatorname{Ad}(\bar{v}) \circ \bar{\beta}_{\{M\}} \circ \eta_{\mathfrak{e}_{\mathfrak{A}}} \circ\left(\iota_{\mathfrak{A}, X} \circ j_{\mathbf{\square}}+\iota_{\mathfrak{A}, X_{\square} \backslash\{M\}} \circ j_{\square}\right)=\eta_{\mathfrak{e}_{\mathfrak{B}}} \circ\left(\psi \circ j \mathbf{\square}+\beta_{X_{\square} \backslash\{M\}} \circ j_{\square}\right)$.

Note that the Busby invariant of the extension

$$
0 \rightarrow \mathfrak{A}(\{M\}) \rightarrow \mathcal{E}_{1} \rightarrow\left(p_{1}+p_{2}\right)\left(\mathfrak{A}\left(X_{\square}\right) \oplus \mathfrak{A}\left(X_{\square} \backslash\{M\}\right)\right)\left(p_{1}+p_{2}\right) \rightarrow 0
$$

is given by $\eta_{\mathfrak{e}_{\mathfrak{A}}} \circ\left(\iota_{\mathfrak{A}, X} \circ j \mathbf{\square}+\iota_{\mathfrak{A}, X_{\square} \backslash\{M\}} \circ j_{\square}\right)$ and the Busby invariant of the extension

$$
0 \rightarrow \mathfrak{B}(\{M\}) \rightarrow \mathcal{E}_{2} \rightarrow\left(e_{1}+e_{2}\right)\left(\mathfrak{B}\left(X_{\mathbf{\square}}\right) \oplus \mathfrak{B}\left(X_{\square} \backslash\{M\}\right)\right)\left(e_{1}+e_{2}\right) \rightarrow 0
$$

is given by $\eta_{\mathfrak{e}_{\mathfrak{B}}} \circ\left(\kappa_{\square}+\kappa_{\square}\right)$, where $\kappa_{\square}: e_{1} \mathfrak{B}\left(X_{\mathbf{\square}}\right) e_{1} \rightarrow \mathfrak{B}\left(X_{\square}\right)$ and $\kappa_{\square}: e_{2} \mathfrak{B}\left(X_{\square} \backslash\right.$ $\{M\}) e_{2} \rightarrow \mathfrak{B}\left(X_{\square} \backslash\{M\}\right)$ are the natural embeddings. Hence, by Equation (11), Theorem 2.2 of [ELP99], and the five lemma, $\mathcal{E}_{1} \cong \mathcal{E}_{2}$. By Lemma 3.13, $\mathcal{E}_{1}$ is isomorphic to a full hereditary sub- $C^{*}$-algebra of $\mathfrak{A}$ and $\mathcal{E}_{2}$ is isomorphic to a full hereditary sub- $C^{*}$-algebra of $\mathfrak{B}$. We have just proved the claim.

If $X_{\square}=\emptyset$ the result is due to Elliott's classification result [Ell76], and if $X_{\square} \backslash$ $\{M\}=\emptyset$ the theorem follows easily by making modifications to the above proof.

Remark 3.16. Let $\mathfrak{A}$ and $\mathfrak{B}$ be graph $C^{*}$-algebras satisfying Condition $(\mathrm{K})$ that are $C^{*}$-algebras over $X$ such that each of $\mathfrak{A}\left(X_{i}\right), \mathfrak{B}\left(X_{i}\right)$ are either $A F$ algebras or $\mathcal{O}_{\infty^{-}}$ absorbing and such that $\mathfrak{A}\left(X_{i}\right)$ and $\mathfrak{B}\left(X_{i}\right)$ are tight $C^{*}$-algebras over $X_{i}$, whenever $\mathfrak{A}\left(X_{i}\right)$ and $\mathfrak{B}\left(X_{i}\right)$ are $\mathcal{O}_{\infty}$-absorbing. Assume that there exists an isomorphism $\alpha: \mathrm{FK}_{X}^{+}(\mathfrak{A}) \rightarrow \mathrm{FK}_{X}^{+}(\mathfrak{B})$. Assume moreover, that $\mathfrak{A}(\{M\})$ is an $A F$ algebra and that for every ideal $\mathfrak{I}$ of $\mathfrak{A}$, we have that $\mathfrak{I} \subseteq \mathfrak{A}(\{M\})$ or $\mathfrak{A}(\{M\}) \subseteq \mathfrak{I}$. Then $\mathfrak{A} \otimes \mathbb{K} \cong \mathfrak{B} \otimes \mathbb{K}$. This follows from the proof above together with Corollary 5.6 of ERRa and applies to the cases]

(a) 4.1E.4 and 4.1E.C, where we view the algebra $\mathfrak{A}$ that is tight over the space 4.1E as a $C^{*}$-algebra over $a \rightarrow b \leftarrow c$ as indicated by the assignment

(b) 4.1F.4 and 4.1F.C, where we view the algebra $\mathfrak{A}$ that is tight over the space $4.1 \mathrm{~F}$ as a $C^{*}$-algebra over $a \rightarrow b \leftarrow c$ as indicated by the assignment

The following result resolves the cases 3.3.2, 3.3.3, 4.A.1, 4.A.3, 4.A.7.

Theorem 3.17. Let $\mathfrak{A}$ and $\mathfrak{B}$ be graph $C^{*}$-algebras that are tight $C^{*}$-algebras over $X$, with $X_{i}$ being a singleton, for each $i=1,2, \ldots, n$. Suppose there exists an isomorphism $\alpha: \mathrm{FK}_{X}^{+}(\mathfrak{A}) \rightarrow \mathrm{FK}_{X}^{+}(\mathfrak{B})$ such that $\alpha$ lifts to an invertible element in $K K(X ; \mathfrak{A}, \mathfrak{B})$. Then $\mathfrak{A} \otimes \mathbb{K} \cong \mathfrak{B} \otimes \mathbb{K}$.

Proof. Note that we may assume that $\mathfrak{A}$ and $\mathfrak{B}$ are stable $C^{*}$-algebras. If $\mathfrak{A}(\{M\})$ is an $A F$ algebra, then the theorem follows from Theorem 3.15. Suppose $\mathfrak{A}(\{M\})$

\footnotetext{
${ }^{4}$ Here we specify how we view the algebras as algebras over $a \rightarrow b \leftarrow c$ by providing a continuous map from the primitive ideal space to $\{a, b, c\}$
} 
is $\mathcal{O}_{\infty}$-absorbing. Then $\mathfrak{B}(\{M\})$ is $\mathcal{O}_{\infty}$-absorbing. Hence, by Proposition 3.9 and Lemma 3.14, the extensions

$$
\begin{aligned}
0 & \rightarrow \mathfrak{A}(\{M\}) \rightarrow \mathfrak{A} \rightarrow \mathfrak{A}(X \backslash\{M\}) \rightarrow 0, \\
0 & \rightarrow \mathfrak{B}(\{M\}) \rightarrow \mathfrak{B} \rightarrow \mathfrak{B}(X \backslash\{M\}) \rightarrow 0
\end{aligned}
$$

are full extensions. The theorem now follows from the results of Theorem 4.6 of ERRa.

\section{A pullback technique}

The main idea of this section is to write the algebra as a pullback of extensions we can classify coherently. The problem is, that classification usually does not give us unique isomorphisms on the algebra level. But when the quotient is an $A F$ algebra we can in certain cases use that the $K K$-class of the isomorphism is unique. The main idea here is similar to the main idea of Section 3 ,

Lemma 4.1. For each $i=1,2$, let there be given $C^{*}$-algebras $\mathfrak{A}_{i}, \mathfrak{B}_{i}$, and $\mathfrak{C}_{i}$ together with $*$-homomorphisms $\alpha_{i}: \mathfrak{A}_{i} \rightarrow \mathfrak{C}_{i}$ and $\beta_{i}: \mathfrak{B}_{i} \rightarrow \mathfrak{C}_{i}$. Let $\mathfrak{P}_{i}$ denote the pullback of $\mathfrak{A}_{i}$ and $\mathfrak{B}_{i}$ along $\alpha_{i}$ and $\beta_{i}$, for each $i=1,2$.

Assume that there are isomorphisms $\varphi_{\mathfrak{A}}: \mathfrak{A}_{1} \rightarrow \mathfrak{A}_{2}, \varphi_{\mathfrak{B}}: \mathfrak{B}_{1} \rightarrow \mathfrak{B}_{2}$ and $\varphi_{\mathfrak{C}}:$ $\mathfrak{C}_{1} \rightarrow \mathfrak{C}_{2}$, such that the following diagram commutes:

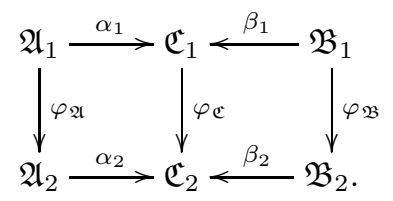

Then we get a canonically induced isomorphism from $\mathfrak{P}_{1}$ to $\mathfrak{P}_{2}$.

Proof. The existence of the $*$-homomorphism from $\mathfrak{P}_{1}$ to $\mathfrak{P}_{2}$ follows from the universal property of the pullback. That this $*$-homomorphism is an isomorphism also follows from the universal property.

Lemma 4.2. Let $\mathfrak{I}$ and $\mathfrak{J}$ be ideals of a $C^{*}$-algebra $\mathfrak{A}$ satisfying $\mathfrak{I} \cap \mathfrak{J}=0$. Then $\mathfrak{A}$ is the pullback of $\mathfrak{A} / \mathfrak{J}$ and $\mathfrak{A} / \mathfrak{I}$ along the quotient maps $\mathfrak{A} / \mathfrak{J} \rightarrow \mathfrak{A} /(\mathfrak{I}+\mathfrak{J})$ and $\mathfrak{A} / \mathfrak{I} \rightarrow \mathfrak{A} /(\mathfrak{I}+\mathfrak{J})$.

Proof. This follows from Proposition 3.1 of Ped99] by noting that we have a commuting diagram

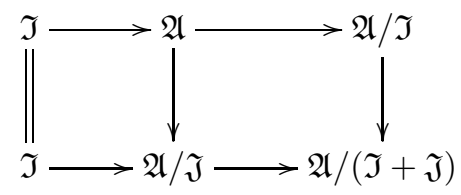

with short exact rows.

The signatures 4.E.4 and 4.E.5 are covered by the following theorem.

Theorem 4.3. Let $\mathfrak{A}$ and $\mathfrak{B}$ be graph $C^{*}$-algebras that are tight over $X$, where $X$ is some finite $T_{0}$ space. Assume that there exists an isomorphism $\alpha: \mathrm{FK}_{X}^{+}(\mathfrak{A}) \rightarrow$ $\mathrm{FK}_{X}^{+}(\mathfrak{B})$. Assume, moreover, that we have disjoint open subsets $O_{0}$ and $O_{1}$ of $X$. Let

$$
Y_{0}=X \backslash O_{1}, \quad Y_{1}=X \backslash O_{0}, \quad \text { and } \quad Z=X \backslash\left(O_{0} \cup O_{1}\right) .
$$


Assume also $Z \neq \emptyset$ and that $\mathfrak{A}(Z)$ is an $A F$ algebra.

For each $i=0,1$, if $\mathfrak{A}\left(O_{i}\right)$ is $\mathcal{O}_{\infty}$-absorbing, then we assume that:

(a) There exist two disjoint clopen subsets $Y_{i}^{1}$ and $Y_{i}^{2}$ of $Y_{i}$ (with the subspace topology) such that $Y_{i}=Y_{i}^{1} \cup Y_{i}^{2}$ and $O_{i} \subseteq Y_{i}^{1}$.

(b) The ideal lattice of $\mathfrak{A}\left(O_{i}\right)$ is linear, i.e., $O_{i} \cong \mathrm{X}_{j}$ for some $j$.

(c) $\mathfrak{A}\left(O_{i}\right)$ is an essential ideal of $\mathfrak{A}\left(Y_{i}^{1}\right)$

(d) $\mathfrak{A}\left(\left\{m_{i}\right\}\right)$ is essential in $\mathfrak{A}\left(\left\{m_{i}\right\} \cup\left(Y_{i}^{1} \backslash O_{i}\right)\right)$, where $m_{i}$ is the least element of $\mathrm{O}_{i}$.

Then $\mathfrak{A} \otimes \mathbb{K} \cong \mathfrak{B} \otimes \mathbb{K}$.

Proof. We may assume that $\mathfrak{A}$ and $\mathfrak{B}$ are stable $C^{*}$-algebras. Note that for each locally closed subset $Y$ of $X, \mathfrak{A}(Y)$ is an $A F$ algebra if and only if $\mathfrak{B}(Y)$ is an $A F$ algebra, and $\mathfrak{A}(Y)$ is $\mathcal{O}_{\infty}$-absorbing if and only if $\mathfrak{B}(Y)$ is $\mathcal{O}_{\infty}$-absorbing (since there exists a positive isomorphism from $K_{0}(\mathfrak{A}(Y))$ to $\left.K_{0}(\mathfrak{B}(Y))\right)$. Specifically $\mathfrak{B}\left(X \backslash\left(O_{0} \cup O_{1}\right)\right)$ is an $A F$ algebra.

Note that the diagram

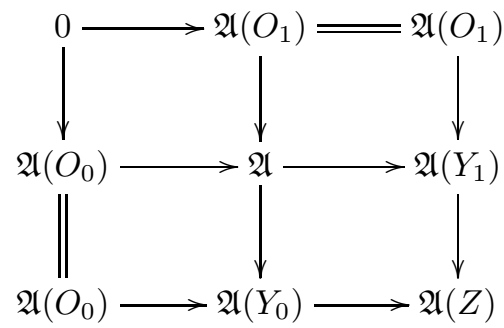

is commutative with short exact rows and columns, analogously for $\mathfrak{B}$.

If both $\mathfrak{A}\left(O_{0}\right)$ and $\mathfrak{A}\left(O_{1}\right)$ are $A F$ algebras, then it follows from the permanence properties of $A F$ algebras that $\mathfrak{A}$ is an $A F$ algebra, and thus also $\mathfrak{B}$. In this case the theorem follows from Elliott's classification result Ell76.

Now assume that $\mathfrak{A}\left(O_{0}\right)$ is an $A F$ algebra and that $\mathfrak{A}\left(O_{1}\right)$ is $\mathcal{O}_{\infty}$-absorbing. Let $Z_{1}^{1}=Z \backslash Y_{1}^{2}$ and $Z_{1}^{2}=Y_{1}^{2}$. Then $Z_{1}^{1}$ and $Z_{1}^{2}$ are locally closed subsets of $X$, and $Z$ is the disjoint union of $Z_{1}^{1}$ and $Z_{1}^{2}$. Since $\mathfrak{A}\left(Y_{0}\right)$ and $\mathfrak{B}\left(Y_{0}\right)$ are extensions of $A F$ algebras, these are themselves $A F$ algebras. Since $\alpha_{Y_{0}}: K_{0}\left(\mathfrak{A}\left(Y_{0}\right)\right) \rightarrow K_{0}\left(\mathfrak{B}\left(Y_{0}\right)\right)$ is a positive isomorphism, there exists an isomorphism $\beta: \mathfrak{A}\left(Y_{0}\right) \rightarrow \mathfrak{B}\left(Y_{0}\right)$ such that $K_{0}(\beta)=\alpha_{Y_{0}}$ (by Elliott's classification result [Ell76]). Since $\mathfrak{A}\left(Y_{0}\right)$ and $\mathfrak{B}\left(Y_{0}\right)$ are $A F$ algebras and $\beta$ is an $Y_{0}$-equivariant isomorphism, we have that $K_{0}\left(\beta_{Y}\right)=\alpha_{Y}$ for all $Y \in \mathbb{L} \mathbb{C}(X)$ such that $Y \subseteq Y_{0}$.

Let

$$
\mathfrak{e}^{\mathfrak{A}}: 0 \rightarrow \mathfrak{A}\left(O_{1}\right) \rightarrow \mathfrak{A}\left(Y_{1}^{1}\right) \rightarrow \mathfrak{A}\left(Z^{1}\right) \rightarrow 0 .
$$

and

$$
\mathfrak{e}^{\mathfrak{B}}: 0 \rightarrow \mathfrak{B}\left(O_{1}\right) \rightarrow \mathfrak{B}\left(Y_{1}^{1}\right) \rightarrow \mathfrak{B}\left(Z^{1}\right) \rightarrow 0 .
$$

Since $\alpha: \mathrm{FK}_{X}^{+}(\mathfrak{A}) \rightarrow \mathrm{FK}_{X}^{+}(\mathfrak{B})$ is an isomorphism, we also have an isomorphism $\alpha_{Y_{1}^{1}}: \mathrm{FK}_{Y_{1}^{1}}^{+}(\mathfrak{A}) \rightarrow \mathrm{FK}_{Y_{1}^{1}}^{+}(\mathfrak{B})$. So by Theorem 4.14 of of [MNa, Kirchberg [Kir00], and Theorem 3.3 of [ERRa], there exists an isomorphism $\varphi: \mathfrak{A}\left(O_{1}\right) \rightarrow \mathfrak{B}\left(O_{1}\right)$ such that $K_{*}(\varphi)=\alpha_{O_{1}}$, and

$$
\left[\eta_{\mathfrak{e}^{\mathfrak{B}}} \circ \beta_{O_{Z^{1}}}\right]=\left[\bar{\varphi} \circ \eta_{\mathfrak{e}^{\mathfrak{A l}}}\right]
$$

in $K K^{1}\left(\mathfrak{A}\left(Z^{1}\right), \mathfrak{B}\left(O_{1}\right)\right)$, since $K K\left(\beta_{Z^{1}}\right)$ is the unique lifting of $\alpha_{Z^{1}}$. 
As in the proof of Proposition 6.3 of [ERRa, Corollary 5.3 of [ERRa] implies that $\eta_{\mathfrak{e}^{\mathfrak{A}}}$ and $\eta_{\mathfrak{e}^{\mathfrak{B}}}$ are full extensions, and thus also the extensions with Busby maps $\eta_{\mathfrak{e}^{\mathfrak{B}}} \circ \beta_{Z^{1}}$ and $\bar{\varphi} \circ \eta_{\mathfrak{e}^{\mathfrak{A}}}$ are full. Since the extensions are non-unital and $\mathfrak{B}\left(O_{1}\right)$ satisfies the corona factorization property, there exists a unitary $u \in \mathcal{M}\left(\mathfrak{B}\left(O_{1}\right)\right)$ such that

$$
\eta_{\mathfrak{e}^{\mathfrak{B}}} \circ \beta_{Z^{1}}=\operatorname{Ad}(\bar{u}) \circ \bar{\varphi} \circ \eta_{\mathfrak{e}^{\mathfrak{A}}}
$$

where $\bar{u}$ is the image of $u$ in the corona algebra (this follows from EK01 and KN06]). Hence, by Theorem 2.2 of [ELP99, there exists an isomorphism $\eta$ : $\mathfrak{A}\left(Y_{1}^{1}\right) \rightarrow \mathfrak{B}\left(Y_{1}^{1}\right)$ such that $\left(\operatorname{Ad}(\bar{u}) \circ \varphi, \eta, \beta_{Z_{1}^{1}}\right)$ is an isomorphism from $\mathfrak{e}^{\mathfrak{A}}$ to $\mathfrak{e}^{\mathfrak{B}}$.

Since the extension

$$
0 \rightarrow \mathfrak{A}\left(O_{1}\right) \rightarrow \mathfrak{A}\left(Y_{1}\right) \rightarrow \mathfrak{A}(Z) \rightarrow 0
$$

is the direct sum of the extensions

$$
0 \rightarrow \mathfrak{A}\left(O_{1}\right) \rightarrow \mathfrak{A}\left(Y_{1}^{1}\right) \rightarrow \mathfrak{A}\left(Z^{1}\right) \rightarrow 0
$$

and

$$
0 \rightarrow 0 \rightarrow \mathfrak{A}\left(Z^{2}\right) \rightarrow \mathfrak{A}\left(Z^{2}\right) \rightarrow 0
$$

and analogously for $\mathfrak{B}$, we get an isomorphism from $0 \rightarrow \mathfrak{A}\left(O_{1}\right) \rightarrow \mathfrak{A}\left(Y_{1}\right) \rightarrow$ $\mathfrak{A}(Z) \rightarrow 0$ to $0 \rightarrow \mathfrak{B}\left(O_{1}\right) \rightarrow \mathfrak{B}\left(Y_{1}\right) \rightarrow \mathfrak{B}(Z) \rightarrow 0$, which is equal to $\beta_{Z}$ on the quotient. Now the theorem follows from Lemma 4.2 and Lemma 4.1 .

Now assume instead that both $\mathfrak{I}$ and $\mathfrak{J}$ are $\mathcal{O}_{\infty}$-absorbing. The proof is similar to the case above. Instead of lifting $\alpha_{Y_{0}}: K_{0}\left(\mathfrak{A}\left(Y_{0}\right)\right) \rightarrow K_{0}\left(\mathfrak{B}\left(Y_{0}\right)\right)$ to $\beta: \mathfrak{A}\left(Y_{0}\right) \rightarrow$ $\mathfrak{B}\left(Y_{0}\right)$ we just lift $\alpha_{Z}: K_{0}(\mathfrak{A}(Z)) \rightarrow K_{0}(\mathfrak{B}(Z))$ to $\beta: \mathfrak{A}(Z) \rightarrow \mathfrak{B}(Z)$. Then we do as above first for the extensions corresponding to the relative open subset $O_{0}$ of $Y_{0}$ and then for the extensions corresponding to the relative open subset $O_{1}$ of $Y_{1}$. As above, the theorem then follows from Lemma 4.2 and Lemma 4.1.

\section{AD HOC METHODS}

In this section we present arguments which resolve the classification question for some examples of tempered ideal spaces which are not covered by the general results above. Most of the results are based on knowing strong classification for smaller ideal spaces, as explained below. Our results of this nature, presented in ERRc, are of a rather limited scope, and require restrictions on the $K$-theory, requiring the $K$-groups to be finitely generated, or even for the graph $C^{*}$-algebra to be unital. We will see this idea in use in a very clear form in the two open cases for three primitive ideals (cf. Section 5.1) and in more complicated four-point cases.

Our starting point is

Theorem 5.1. Let $\mathfrak{A}_{1}$ and $\mathfrak{A}_{2}$ be graph $C^{*}$-algebras that are tight $C^{*}$-algebras over a finite $T_{0}$-space $X$ and let $U \in \mathbb{O}(X)$ be non-empty. Let $\mathfrak{e}_{i}$ be the extension $0 \rightarrow \mathfrak{A}_{i}(U) \otimes \mathbb{K} \rightarrow \mathfrak{A}_{i} \otimes \mathbb{K} \rightarrow \mathfrak{A}_{i}(X \backslash U) \otimes \mathbb{K} \rightarrow 0$. Suppose

(1) $\mathfrak{e}_{i}$ is a full extension;

(2) there exists an invertible element $\alpha \in K K\left(X ; \mathfrak{A}_{1}, \mathfrak{A}_{2}\right)$; and

(3) the induced invertible element $\alpha_{Y} \in K K\left(\mathfrak{A}_{1}(Y) \otimes \mathbb{K}, \mathfrak{A}_{2}(Y) \otimes \mathbb{K}\right)$ lifts to an isomorphism from $\mathfrak{A}_{1}(Y) \otimes \mathbb{K}$ to $\mathfrak{A}_{2}(Y) \otimes \mathbb{K}$ for $Y=U$ and $Y=X \backslash U$.

Then $\mathfrak{A}_{1} \otimes \mathbb{K} \cong \mathfrak{A}_{2} \otimes \mathbb{K}$. 
Proof. By (3), there exists an isomorphism $\varphi_{Y}: \mathfrak{A}_{1}(Y) \otimes \mathbb{K} \rightarrow \mathfrak{A}_{2}(Y) \otimes \mathbb{K}$ for $Y=U$ and $Y=X \backslash U$ such that $K K\left(\varphi_{Y}\right)=\alpha_{Y}$. It follows from (1) that $\mathfrak{e}_{i}$ are essential, so by [ERRa, Theorem 3.3], $\alpha_{X \backslash U} \times\left[\eta_{\mathfrak{e}_{2}}\right]=\left[\eta_{\mathfrak{e}_{1}}\right] \times \alpha_{U}$. Therefore, $K K\left(\varphi_{X \backslash U}\right) \times\left[\eta_{\mathfrak{e}_{2}}\right]=\left[\eta_{\mathfrak{e}_{1}}\right] \times K K\left(\varphi_{U}\right)$. Hence, by [ERRa, Proposition 6.1 and Lemma 4.5], we have that $\mathfrak{A}_{1} \otimes \mathbb{K} \cong \mathfrak{A}_{2} \otimes \mathbb{K}$.

Definition 5.2. For a $T_{0}$ topological space $X$, we will consider classes $\mathcal{C}_{X}$ of separable, nuclear $C^{*}$-algebras in the bootstrap category of Rosenberg and Schochet $\mathcal{N}$ such that

(1) any element in $\mathcal{C}_{X}$ is a $C^{*}$-algebra over $X$;

(2) if $\mathfrak{A}$ and $\mathfrak{B}$ are in $\mathcal{C}_{X}$ and there exists an invertible element $\alpha$ in $K K(X ; \mathfrak{A}, \mathfrak{B})$ which induces an isomorphism from $\mathrm{FK}_{X}^{+}(\mathfrak{A})$ to $\mathrm{FK}_{X}^{+}(\mathfrak{B})$, then there exists an isomorphism $\varphi: \mathfrak{A} \rightarrow \mathfrak{B}$ such that $K K(\varphi)=\alpha_{X}$, where $\alpha_{X}$ is the element in $K K(\mathfrak{A}, \mathfrak{B})$ induced by $\alpha$.

Remark 5.3. Let $X$ be a finite $T_{0}$-space, let $U$ be an open subset of $X$, and let $\mathcal{C}_{U}$ and $\mathcal{C}_{X \backslash U}$ be classes of $C^{*}$-algebras satisfying the conditions of Definition 5.2 If $\mathfrak{A}_{1}$ and $\mathfrak{A}_{2}$ are separable $C^{*}$-algebras such that $\mathfrak{A}_{1}(U), \mathfrak{A}_{2}(U) \in \mathcal{C}_{U}$ and $\mathfrak{A}_{1}(X \backslash$ $U), \mathfrak{A}_{2}(X \backslash U) \in \mathcal{C}_{X \backslash U}$, then (3) in Theorem 5.11 holds.

Let $\mathcal{C}_{X}$ and $\mathcal{C}_{Y}$ be classes of $C^{*}$-algebras satisfy the conditions in Definition 5.2 . Let $\mathcal{C}_{X \sqcup Y}$ be the classes of $C^{*}$-algebras consisting of elements $\mathfrak{A} \oplus \mathfrak{B}$ with $\mathfrak{A} \in \mathcal{C}_{X}$ and $\mathfrak{B} \in \mathcal{C}_{Y}$. Then $\mathcal{C}_{X \sqcup Y}$ satisfies the conditions in Definition 5.2

Remark 5.4. Here we will provide some examples of classes satisfying the conditions in Definition 5.2

(1) By [Kir00, the class all stable, nuclear, separable, $\mathcal{O}_{\infty}$-absorbing $C^{*}$-algebras that are tight over a finite $T_{0}$-space satisfy the conditions in Definition 5.2 .

By [ERRc, Corollary 3.10 and Theorem 3.13] and by the results of [EK], the following classes of $C^{*}$-algebras satisfies the conditions in Definition 5.2 .

(2) Let $\mathcal{C}_{\mathbf{X}_{n}}$ be the class of nuclear, separable, tight $C^{*}$-algebras $\mathfrak{A}$ over $\mathbf{X}_{n}$ such that $\mathfrak{A}$ is stable, $\mathfrak{A}(\{n\})$ is a Kirchberg algebra, $\mathfrak{A}([1, n-1])$ is an AF-algebra, and $K_{i}(\mathfrak{A}[Y])$ is finitely generated for all $Y \in \mathbb{L} \mathbb{C}\left(\mathrm{X}_{n}\right)$.

(3) Let $\mathcal{C}_{\mathrm{X}_{2}}^{\prime}$ be the class of unital graph $C^{*}$-algebras with exactly one non-trivial ideal with the ideal being an $A F$ algebra and the quotient $\mathcal{O}_{\infty}$-absorbing, simple $C^{*}$-algebras. Let $\mathcal{C}_{\mathrm{X}_{2}}$ be the class of $C^{*}$-algebras $\mathfrak{A}$ such that $\mathfrak{A} \cong \mathfrak{B} \otimes \mathbb{K}$ for some $\mathfrak{B} \in \mathcal{C}_{\mathrm{X}_{2}}^{\prime}$.

By [Ell76], the following class of $C^{*}$-algebras satisfy the conditions in Definition 5.2 ,

(4) Let $\mathcal{C}_{X}$ be the class of stable AF-algebras over $X$.

5.1. Linear spaces. This case is solved in ERRc, and the reader is referred there for details. However, since this is the most basic case in which our approach via Theorem 5.1 is applied, we will explain the methods for the benefit of the reader.

Lemma 5.5. Let $\mathfrak{A}$ be a graph $C^{*}$-algebra such that $\mathfrak{A}$ is a tight $C^{*}$-algebra over $\mathrm{X}_{n}$.

(i) If $\mathfrak{A}(\{n\})$ and $\mathfrak{A}(\{1\})$ are $\mathcal{O}_{\infty}$-absorbing and $\mathfrak{A}([2, n-1])$ is an AF-algebra, then

$$
\mathfrak{e}: 0 \rightarrow \mathfrak{A}([2, n]) \otimes \mathbb{K} \rightarrow \mathfrak{A} \otimes \mathbb{K} \rightarrow \mathfrak{A}(\{1\}) \otimes \mathbb{K} \rightarrow 0
$$


is a full extension.

(ii) If $\mathfrak{A}([k, n])$ and $\mathfrak{A}([1, k-2])$ are $A F$-algebras and $\mathfrak{A}(\{k-1\})$ is $\mathcal{O}_{\infty}$-absorbing, then

$$
\mathfrak{e}: 0 \rightarrow \mathfrak{A}([k, n]) \otimes \mathbb{K} \rightarrow \mathfrak{A} \otimes \mathbb{K} \rightarrow \mathfrak{A}([1, k-1]) \otimes \mathbb{K} \rightarrow 0
$$

is a full extension.

(iii) If $\mathfrak{A}([k, n])$ and $\mathfrak{A}([1, k-2])$ are AF-algebras and $\mathfrak{A}(\{k-1\})$ is $\mathcal{O}_{\infty}$-absorbing, then

$$
\mathfrak{e}: 0 \rightarrow \mathfrak{A}([k-1, n]) \otimes \mathbb{K} \rightarrow \mathfrak{A} \otimes \mathbb{K} \rightarrow \mathfrak{A}([1, k-2]) \otimes \mathbb{K} \rightarrow 0
$$

is a full extension.

Proof. In ERRc, we prove (i) and (ii). We now prove (iii). Note that

$$
0 \rightarrow \mathfrak{A}(\{k-1\}) \otimes \mathbb{K} \rightarrow \mathfrak{A}([k-2, k-1]) \otimes \mathbb{K} \rightarrow \mathfrak{A}(\{k-2\}) \otimes \mathbb{K} \rightarrow 0
$$

is full since this is an essential extension and $\mathfrak{A}(\{k-1\})$ is $\mathcal{O}_{\infty}$-absorbing. Since $\mathfrak{A}([k, n])$ is the largest $A F$-ideal of $\mathfrak{A}([k-1, n])$ and $\mathfrak{A}([k-1, n]) / \mathfrak{A}([k, n])=\mathfrak{A}(\{k-$ $1\})$ is $\mathcal{O}_{\infty}$-absorbing, by [ET10, Proposition 3.10] and [ERR09, Lemma 1.5], $0 \rightarrow$ $\mathfrak{A}([k, n]) \otimes \mathbb{K} \rightarrow \mathfrak{A}([k-1, n]) \otimes \mathbb{K} \rightarrow \mathfrak{A}(\{k-1\}) \otimes \mathbb{K} \rightarrow 0$ is full. By ERR10, Proposition 3.2], $0 \rightarrow \mathfrak{A}([k-1, n]) \otimes \mathbb{K} \rightarrow \mathfrak{A}([k-2, n]) \otimes \mathbb{K} \rightarrow \mathfrak{A}(\{k-2\}) \otimes \mathbb{K} \rightarrow 0$ is full. Since $\mathfrak{A}(\{k-2\})=\mathfrak{A}([k-2, n]) / \mathfrak{A}([k-1, n])$ is an essential of $\mathfrak{A} / \mathfrak{A}([k-1, n])$, the extension in (iii) is full by [ERRa, Proposition 5.4].

To solve the cases 3.7.5 and 4.3F.9, we now argue as follows:

Theorem 5.6. Let $\mathfrak{A}_{1}$ and $\mathfrak{A}_{2}$ be graph $C^{*}$-algebras that are tight $C^{*}$-algebras over $\mathrm{X}_{n}$. Suppose

(i) $\mathfrak{A}_{i}(\{n\})$ and $\mathfrak{A}_{i}(\{1\})$ are $\mathcal{O}_{\infty}$-absorbing;

(ii) $\mathfrak{A}_{i}([2, n-1])$ is an AF-algebra; and

(iii) the K-groups of $\mathfrak{A}_{i}$ are finitely generated.

Then $\mathfrak{A}_{1} \otimes \mathbb{K} \cong \mathfrak{A}_{2} \otimes \mathbb{K}$ if and only if $\mathrm{FK}_{\mathbf{X}_{n}}^{+}\left(\mathfrak{A}_{1} \otimes \mathbb{K}\right) \cong \mathrm{FK}_{\mathbf{X}_{n}}^{+}\left(\mathfrak{A}_{2} \otimes \mathbb{K}\right)$.

Proof. Let $\mathfrak{e}_{i}$ be the extension

$$
0 \rightarrow \mathfrak{A}_{i}([2, n]) \otimes \mathbb{K} \rightarrow \mathfrak{A}_{i} \otimes \mathbb{K} \rightarrow \mathfrak{A}_{i}(\{1\}) \otimes \mathbb{K} \rightarrow 0 .
$$

By Lemma 5.5(i), $\mathfrak{e}_{i}$ is a full extension. Thus, Assumption (1) of Theorem 5.1 holds. Suppose $\alpha$ : $\mathrm{FK}_{\mathbf{X}_{n}}^{+}\left(\mathfrak{A}_{1} \otimes \mathbb{K}\right) \rightarrow \mathrm{FK}_{\mathbf{X}_{n}}^{+}\left(\mathfrak{A}_{2} \otimes \mathbb{K}\right)$ is an isomorphism. Lift $\alpha$ to an invertible element $x \in K K\left(\mathrm{X}_{n} ; \mathfrak{A}_{1} \otimes \mathbb{K}, \mathfrak{A}_{2} \otimes \mathbb{K}\right)$, such a lifting exists by Theorem 4.14 of [MNa]. Therefore, Assumption (2) of Theorem 5.1 holds.

Note now that $x$ induces invertible elements $r_{\mathbf{X}_{n}}^{[2, n]}(x)$ in $K K\left([2, n] ; \mathfrak{A}_{1}([2, n]) \otimes\right.$ $\left.\mathbb{K}, \mathfrak{A}_{2}([2, n]) \otimes \mathbb{K}\right)$ and $r_{\mathbf{X}_{n}}^{[1]}(x)$ in $K K\left(\mathfrak{A}(\{1\}) \otimes \mathbb{K}, \mathfrak{A}_{2}(\{1\}) \otimes \mathbb{K}\right)$. Note that $\mathfrak{A}_{i}([2, n])$ has a smallest ideal $\mathfrak{A}_{i}(\{n\})$ which is $\mathcal{O}_{\infty}$-absorbing and the quotient $\mathfrak{A}_{i}([2, n-$ 1]) is an $A F$ algebra. By Theorem 3.9 of [ERRc, there exists an isomorphism $\varphi: \mathfrak{A}_{1}([2, n]) \otimes \mathbb{K} \rightarrow \mathfrak{A}_{2}([2, n]) \otimes \mathbb{K}$ such that $K L(\varphi)$ is the invertible element in $K L\left(\mathfrak{A}_{1}([2, n]), \mathfrak{A}_{2}([2, n])\right)$ induced by $x$. Since the $K$-theory of $\mathfrak{A}_{1}$ is finitely generated, $K L\left(\mathfrak{A}_{1}([2, n]), \mathfrak{A}_{2}([2, n])\right)=K K\left(\mathfrak{A}_{1}([2, n]), \mathfrak{A}_{2}([2, n])\right)$. Thus, $K K(\varphi)$ is the invertible element in $K K\left(\mathfrak{A}_{1}([2, n]), \mathfrak{A}_{2}([2, n])\right)$ induced by $x$. By the KirchbergPhillips classification, there exists an isomorphism $\psi: \mathfrak{A}_{1}(\{1\}) \otimes \mathbb{K} \rightarrow \mathfrak{A}_{2}(\{1\}) \otimes \mathbb{K}$ lifting $r_{\mathbf{X}_{n}}^{[1]}(x)$. We have just shown that Assumption (3) of Theorem 5.1 holds.

By Theorem 5.1, we can conclude that $\mathfrak{A}_{1} \otimes \mathbb{K} \cong \mathfrak{A}_{2} \otimes \mathbb{K}$. 
Similarly, one solves 3.7.2, 4.3F.2, and 4.3F.4 using

Theorem 5.7. Let $\mathfrak{A}_{1}$ and $\mathfrak{A}_{2}$ be graph $C^{*}$-algebras that are tight $C^{*}$-algebras over $\mathrm{X}_{n}$. Suppose

(i) $\mathfrak{A}_{i}([k, n])$ and $\mathfrak{A}_{i}([1, k-2])$ are $A F$ algebras;

(ii) $\mathfrak{A}_{i}(\{k-1\})$ is $\mathcal{O}_{\infty}$-absorbing; and

(iii) the $K$-groups of $\mathfrak{A}_{i}$ are finitely generated.

Then $\mathfrak{A}_{1} \otimes \mathbb{K} \cong \mathfrak{A}_{2} \otimes \mathbb{K}$ if and only if $\mathrm{FK}_{\mathrm{X}_{n}}^{+}\left(\mathfrak{A}_{1} \otimes \mathbb{K}\right) \cong \mathrm{FK}_{\mathrm{X}_{n}}^{+}\left(\mathfrak{A}_{2} \otimes \mathbb{K}\right)$.

A proof is given in ERRc.

\subsection{Accordion spaces.}

Lemma 5.8. Let $\mathfrak{A}$ be a graph $C^{*}$-algebra with signature 4.F.x, and let $\mathfrak{I}$ be the smallest ideal of $\mathfrak{A}$.

(1) When $x=3,5,7,9, A, B, D$, then the extension $0 \rightarrow \mathfrak{I} \otimes \mathbb{K} \rightarrow \mathfrak{A} \otimes \mathbb{K} \rightarrow \mathfrak{A} / \mathfrak{I} \otimes \mathbb{K} \rightarrow$ 0 is full.

(2) When $x=2,4$, C, then the extension $0 \rightarrow \mathfrak{I} \otimes \mathbb{K} \rightarrow \mathfrak{A} \otimes \mathbb{K} \rightarrow \mathfrak{A} / \mathfrak{I} \otimes \mathbb{K} \rightarrow 0$ is full provided that $\mathfrak{A}$ is unital.

Proof. First note that the extension $0 \rightarrow \mathfrak{I} \otimes \mathbb{K} \rightarrow \mathfrak{A} \otimes \mathbb{K} \rightarrow \mathfrak{A} / \mathfrak{I} \otimes \mathbb{K} \rightarrow 0$ is essential. Hence, in the case 4.F. $\mathrm{x}$ for $\mathrm{x}=3,5,7,9, \mathrm{~B}, \mathrm{D}$ the extension is full since $\mathfrak{I} \otimes \mathbb{K}$ is a simple, purely infinite, stable $C^{*}$-algebra, which implies that $\mathcal{Q}(\mathfrak{I} \otimes \mathbb{K})$ is simple. If $A$ is unital and $Y$ is the space 4.F.x for $\mathrm{x}=2,4$, and C, then the extension is full since in this case $\mathfrak{I} \cong \mathbb{K}$ and $\mathcal{Q}(\mathbb{K})$ is simple. We are left with showing the extension is full for the case 4.F.A. This case follows from ERRa, Proposition 5.4 and Corollary 5.6].

Lemma 5.9. Let $\mathfrak{A}$ be a graph $C^{*}$-algebra with tempered signature 4.3F.x for $x=$ $5,6, A, D$. Then the ideal lattice of $\mathfrak{A}$ is $0 \unlhd \mathfrak{I}_{1} \unlhd \mathfrak{I}_{2} \unlhd \mathfrak{I}_{3} \unlhd \mathfrak{A}$ and the extension $0 \rightarrow \mathfrak{I}_{2} \otimes \mathbb{K} \rightarrow \mathfrak{A} \otimes \mathbb{K} \rightarrow \mathfrak{A} / \mathfrak{I}_{2} \otimes \mathbb{K} \rightarrow 0$ is full.

Proof. We will for show that $\mathfrak{e}: 0 \rightarrow \mathfrak{I}_{2} \otimes \mathbb{K} \rightarrow \mathfrak{I}_{3} \otimes \mathbb{K} \rightarrow \mathfrak{I}_{3} / \mathfrak{I}_{2} \otimes \mathbb{K} \rightarrow 0$ is a full extension. By Lemma 5.5, $\mathfrak{e}$ is a full extension for $x=5, \mathrm{~A}, \mathrm{D}$. Consider the case $\mathrm{x}=6$. Note that $\mathfrak{I}_{2}$ and $\mathfrak{I}_{3} / \mathfrak{I}_{1}$ are isomorphic to non- $A F$ graph $C^{*}$-algebras with exactly one nontrivial ideal. Therefore, by Proposition 3.9.

$$
\begin{aligned}
& 0 \rightarrow \mathfrak{I}_{1} \otimes \mathbb{K} \rightarrow \mathfrak{I}_{2} \otimes \mathbb{K} \rightarrow \mathfrak{I}_{2} / \mathfrak{I}_{1} \otimes \mathbb{K} \rightarrow 0 \\
& 0 \rightarrow \mathfrak{I}_{2} / \mathfrak{I}_{1} \otimes \mathbb{K} \rightarrow \mathfrak{I}_{3} / \mathfrak{I}_{1} \otimes \mathbb{K} \rightarrow \mathfrak{I}_{3} / \mathfrak{I}_{2} \otimes \mathbb{K} \rightarrow 0
\end{aligned}
$$

are full extensions. By [ERR10, Proposition 3.2], $\mathfrak{e}$ is a full extension. The lemma now follows from ERRa, Proposition 5.4].

Lemma 5.10. Let $\mathfrak{A}$ be a graph $C^{*}$-algebra with tempered signature 4.39.x for $x=2,6,9, A, B, C, D$, or $E$. Let $\mathfrak{I}$ be the greatest proper ideal of $\mathfrak{A}$.

(1) If $\mathfrak{A}$ is unital, then the extension $0 \rightarrow \mathfrak{I} \otimes \mathbb{K} \rightarrow \mathfrak{A} \otimes \mathbb{K} \rightarrow \mathfrak{A} / \mathfrak{I} \otimes \mathbb{K} \rightarrow 0$ is full.

(2) When $x=9, B, C, D$, the extension $0 \rightarrow \mathfrak{I} \otimes \mathbb{K} \rightarrow \mathfrak{A} \otimes \mathbb{K} \rightarrow \mathfrak{A} / \mathfrak{I} \otimes \mathbb{K} \rightarrow 0$ is full.

Proof. Suppose $\mathfrak{A}$ is unital. Using the general theory of graph $C^{*}$-algebras with this specific ideal structure, we have that $\mathfrak{I}$ is stable. Since $\mathfrak{A} / \mathfrak{I}$ is simple and unital, the conclusion now follows from [ERR09, Lemma 1.5 and Proposition 1.6]. We now prove the extension $0 \rightarrow \mathfrak{I} \otimes \mathbb{K} \rightarrow \mathfrak{A} \otimes \mathbb{K} \rightarrow \mathfrak{A} / \mathfrak{I} \otimes \mathbb{K} \rightarrow 0$ is always full for the spaces 4.39.x with $\mathrm{x}=9, \mathrm{~B}, \mathrm{C}, \mathrm{D}$. Note that $\mathfrak{I}=\mathfrak{I}_{1} \oplus \mathfrak{I}_{2}$ with $\mathfrak{I}_{1}$ simple and 
$\mathfrak{I}_{2}$ a tight $C^{*}$-algebra over $\mathrm{X}_{2}$. By [ERRc, Lemma 4.5] and ERRa, Corollary 5.3 and Corollary 5.6], we have $0 \rightarrow \mathfrak{I}_{2} \otimes \mathbb{K} \rightarrow \mathfrak{A} / \mathfrak{I}_{1} \otimes \mathbb{K} \rightarrow(\mathfrak{A} / \mathfrak{I}) \otimes \mathbb{K} \rightarrow 0$ is full. Since $\mathfrak{A} / \mathfrak{I}_{2} \otimes \mathbb{K}$ is a non- $A F$ graph $C^{*}$-algebra with exactly one nontrivial ideal, the extension $0 \rightarrow \mathfrak{I}_{1} \otimes \mathbb{K} \rightarrow \mathfrak{A} / \mathfrak{I}_{2} \otimes \mathbb{K} \rightarrow \mathfrak{A} / \mathfrak{I} \otimes \mathbb{K} \rightarrow 0$ is a full extension (cf. Proposition [3.9). Thus, by Lemma [3.6] $0 \rightarrow \mathfrak{I} \otimes \mathbb{K} \rightarrow \mathfrak{A} \otimes \mathbb{K} \rightarrow \mathfrak{A} / \mathfrak{I} \otimes \mathbb{K} \rightarrow 0$ is full.

Using the above lemmas and the Universal Coefficient Theorem of Bentmann and Köhler [BK], we get the following cases:

Corollary 5.11. Let $\mathfrak{A}$ and $\mathfrak{B}$ be graph $C^{*}$-algebras that are tight over a finite accordion space $X$. Assume that there exists an isomorphism from $\mathrm{FK}_{X}^{+}(\mathfrak{A})$ to $\mathrm{FK}_{X}^{+}(\mathfrak{B})$. If

(1) $\mathfrak{A}$ and $\mathfrak{B}$ both have tempered signature 4.F.7, 4.F.9, 4.39.B, 4.39.C, or

(2) $\mathfrak{A}$ and $\mathfrak{B}$ both have finitely generated $K$-theory and have tempered signature 4.F.3, 4.F.A, 4.F.B, 4.39.9, 4.39.D, 4.3F.5, 4.3F.D, or

(3) $\mathfrak{A}$ and $\mathfrak{B}$ both are unital and have tempered signature 4.F.2, 4.F.4, 4.F.5, 4.F.C, 4.F.D, 4.39.2, 4.39.6, 4.39.A, 4.39.E, 4.3F.6, 4.3F.A,

then $\mathfrak{A} \otimes \mathbb{K} \cong \mathfrak{B} \otimes \mathbb{K}$.

Proof. By the above lemmas, all the extensions are full. Note that the specified ideal and quotient for each space belongs to classes of $C^{*}$-algebras satisfying the conditions in Definition 5.2. Hence, the result now follows from Theorem 5.1 and the UCT for accordion spaces.

\section{3. $Y$-shaped spaces.}

Lemma 5.12. Let $\mathfrak{A}$ be a graph $C^{*}$-algebra with tempered signature 4.1F.x for $x=2,5,6,7$, or $D$, and let $\mathfrak{I}_{1}$ be the smallest ideal of $\mathfrak{A}$ and let $\mathfrak{I}_{2}$ be the ideal of $\mathfrak{A}$ containing $\mathfrak{I}_{1}$ such that $\mathfrak{I}_{2} / \mathfrak{I}_{1}$ is simple.

(1) When $x=2,6,7$, or $D$, the extension $0 \rightarrow \mathfrak{I}_{2} \otimes \mathbb{K} \rightarrow \mathfrak{A} \otimes \mathbb{K} \rightarrow \mathfrak{A} / \mathfrak{I}_{2} \otimes \mathbb{K} \rightarrow 0$ is full.

(2) When $x=5$, the extension $0 \rightarrow \mathfrak{I}_{2} \otimes \mathbb{K} \rightarrow \mathfrak{A} \otimes \mathbb{K} \rightarrow \mathfrak{A} / \mathfrak{I}_{2} \otimes \mathbb{K} \rightarrow 0$ is full if $\mathfrak{A}$ is unital.

Proof. Let $\mathfrak{J}_{1}$ and $\mathfrak{J}_{2}$ be the maximal ideals of $\mathfrak{A}$ containing $\mathfrak{I}_{2}$. Suppose $\mathrm{x}=2,6,7$, or D. Then, by Lemma 5.5. [ERR10, Proposition 3.2], and [ERRa, Corollary 5.3 and Corollary 5.6], $0 \rightarrow \mathfrak{I}_{2} \otimes \mathbb{K} \rightarrow \mathfrak{J}_{\ell} \otimes \mathbb{K} \rightarrow \mathfrak{J}_{\ell} / \mathfrak{I}_{2} \otimes \mathbb{K} \rightarrow 0$ is full. Hence, by Lemma 3.14, $0 \rightarrow \mathfrak{I}_{2} \otimes \mathbb{K} \rightarrow \mathfrak{A} \otimes \mathbb{K} \rightarrow \mathfrak{A} / \mathfrak{I}_{2} \otimes \mathbb{K} \rightarrow 0$ is full.

Suppose that the signature is $4.1 F .5$ and $\mathfrak{A}$ is unital. Assume that $\mathfrak{J}_{1} / \mathfrak{I}_{2}$ is an AF-algebra and $\mathfrak{J}_{2} / \mathfrak{I}_{2}$ is purely infinite. By Lemma 5.5, $0 \rightarrow \mathfrak{I}_{2} \otimes \mathbb{K} \rightarrow \mathfrak{J}_{2} \otimes \mathbb{K} \rightarrow$ $\mathfrak{J}_{2} / \mathfrak{I}_{2} \otimes \mathbb{K} \rightarrow 0$ is full. Since $\mathfrak{A}$ is a unital graph $C^{*}$-algebra, we have that $\mathfrak{I}_{2} / \mathfrak{I}_{1} \cong \mathbb{K}$. Therefore, $0 \rightarrow \mathfrak{I}_{2} / \mathfrak{I}_{1} \otimes \mathbb{K} \rightarrow \mathfrak{J}_{1} / \mathfrak{I}_{1} \otimes \mathbb{K} \rightarrow \mathfrak{J}_{1} / \mathfrak{I}_{2} \otimes \mathbb{K} \rightarrow 0$ is full. Since $\mathfrak{I}_{2}$ is a stably isomorphic to a non-AF graph $C^{*}$-algebra with exactly one nontrivial ideal, by Proposition [3.9, $0 \rightarrow \mathfrak{I}_{1} \otimes \mathbb{K} \rightarrow \mathfrak{I}_{2} \otimes \mathbb{K} \rightarrow \mathfrak{I}_{2} / \mathfrak{I}_{1} \otimes \mathbb{K} \rightarrow 0$ is full. By [ERR10, Proposition 3.2],

$$
0 \rightarrow \mathfrak{I}_{2} \otimes \mathbb{K} \rightarrow \mathfrak{J}_{1} \otimes \mathbb{K} \rightarrow \mathfrak{J}_{1} / \mathfrak{I}_{2} \otimes \mathbb{K} \rightarrow 0
$$

is full. Hence, by Lemma 3.14, $0 \rightarrow \mathfrak{I}_{2} \otimes \mathbb{K} \rightarrow \mathfrak{A} \otimes \mathbb{K} \rightarrow \mathfrak{A} / \mathfrak{I}_{2} \otimes \mathbb{K} \rightarrow 0$ is full. 
Lemma 5.13. Let $\mathfrak{A}$ be a graph $C^{*}$-algebra with tempered signature 4.3E.x for $x=3,4,5,9, B$, or $D$, and let $\mathfrak{I}_{1}$ and $\mathfrak{I}_{2}$ be the minimal ideals of $\mathfrak{A}$.

(1) When $x=3,4,5, B, D$, the extension $0 \rightarrow\left(\mathfrak{I}_{1} \oplus \mathfrak{I}_{2}\right) \otimes \mathbb{K} \rightarrow \mathfrak{A} \otimes \mathbb{K} \rightarrow \mathfrak{A} /\left(\mathfrak{I}_{1} \oplus\right.$ $\left.\mathfrak{I}_{2}\right) \otimes \mathbb{K} \rightarrow 0$ is a full extension.

(2) When $x=9$, and $\mathfrak{A}$ is unital, then $0 \rightarrow\left(\mathfrak{I}_{1} \oplus \mathfrak{I}_{2}\right) \otimes \mathbb{K} \rightarrow \mathfrak{A} \otimes \mathbb{K} \rightarrow \mathfrak{A} /\left(\mathfrak{I}_{1} \oplus\right.$ $\left.\mathfrak{I}_{2}\right) \otimes \mathbb{K} \rightarrow 0$ is a full extension.

Proof. Suppose $\mathrm{x}=4,5, \mathrm{~B}$, or D. Let $\mathfrak{I}$ be the ideal of $\mathfrak{A}$ containing $\left(\mathfrak{I}_{1} \oplus \mathfrak{I}_{2}\right)$ such that $\mathfrak{I} /\left(\mathfrak{I}_{1} \oplus \mathfrak{I}_{2}\right)$ is simple. Note that push forward extension of the extension $0 \rightarrow\left(\mathfrak{I}_{1} \oplus \mathfrak{I}_{2}\right) \otimes \mathbb{K} \rightarrow \mathfrak{I} \otimes \mathbb{K} \rightarrow \mathfrak{I} /\left(\mathfrak{I}_{1} \oplus \mathfrak{I}_{2}\right) \otimes \mathbb{K} \rightarrow 0$ via the coordinate projection $\left(\mathfrak{I}_{1} \oplus \mathfrak{I}_{2}\right) \rightarrow \mathfrak{I}_{i}$ is a full extension since its isomorphic to a non- $A F$ graph $C^{*}$-algebras with exactly one nontrivial ideal. Therefore, by Lemma 3.6, $0 \rightarrow\left(\mathfrak{I}_{1} \oplus \mathfrak{I}_{2}\right) \otimes \mathbb{K} \rightarrow$ $\mathfrak{I} \otimes \mathbb{K} \rightarrow \mathfrak{I} /\left(\mathfrak{I}_{1} \oplus \mathfrak{I}_{2}\right) \otimes \mathbb{K} \rightarrow 0$ is a full extension. By [ERRa, Proposition 5.4], $0 \rightarrow\left(\mathfrak{I}_{1} \oplus \mathfrak{I}_{2}\right) \otimes \mathbb{K} \rightarrow \mathfrak{A} \otimes \mathbb{K} \rightarrow \mathfrak{A} /\left(\mathfrak{I}_{1} \oplus \mathfrak{I}_{2}\right) \otimes \mathbb{K} \rightarrow 0$ is a full extension since $\mathfrak{I} /\left(\mathfrak{I}_{1} \oplus \mathfrak{I}_{2}\right) \otimes \mathbb{K}$ is an essential ideal of $\mathfrak{A} /\left(\mathfrak{I}_{1} \oplus \mathfrak{I}_{2}\right) \otimes \mathbb{K}$.

We now prove the extension is full for the case $x=3$. Note that in this case $\mathfrak{I}_{1} \otimes \mathbb{K}$ and $\mathfrak{I}_{2} \otimes \mathbb{K}$ are purely infinite, simple $C^{*}$-algebras. Let $\mathfrak{I}$ be the ideal of $\mathfrak{A}$ containing $\left(\mathfrak{I}_{1} \oplus \mathfrak{I}_{2}\right)$ such that $\mathfrak{I} /\left(\mathfrak{I}_{1} \oplus \mathfrak{I}_{2}\right)$ is simple. By Lemma 3.5 and Lemma 3.6, $0 \rightarrow\left(\mathfrak{I}_{1} \oplus \mathfrak{I}_{2}\right) \otimes \mathbb{K} \rightarrow \mathfrak{I} \otimes \mathbb{K} \rightarrow \mathfrak{I} /\left(\mathfrak{I}_{1} \oplus \mathfrak{I}_{2}\right) \otimes \mathbb{K} \rightarrow 0$ is a full extension. The conclusion now follows from [ERRa, Proposition 5.4] since $\mathfrak{I} /\left(\mathfrak{I}_{1} \oplus \mathfrak{I}_{2}\right) \otimes \mathbb{K}$ is an essential ideal of $\mathfrak{A} /\left(\mathfrak{I}_{1} \oplus \mathfrak{I}_{2}\right) \otimes \mathbb{K}$.

Suppose $\mathrm{x}=9$ and $\mathfrak{A}$ is unital. Then $\mathfrak{I}_{i}$ is either $\mathbb{K}$ or a stable, purely infinite, simple $C^{*}$-algebra. Let $\mathfrak{I}$ be the ideal containing $\mathfrak{I}_{1} \oplus \mathfrak{I}_{2}$ such that $\mathfrak{I} /\left(\mathfrak{I}_{1} \oplus \mathfrak{I}_{2}\right)$ is simple. Note that the signature of $\mathfrak{I}$ is 3.6. By Lemma 3.5 the push forward extension of the extension $0 \rightarrow\left(\mathfrak{I}_{1} \oplus \mathfrak{I}_{2}\right) \otimes \mathbb{K} \rightarrow \mathfrak{I} \otimes \mathbb{K} \rightarrow \mathfrak{I} /\left(\mathfrak{I}_{1} \oplus \mathfrak{I}_{2}\right) \otimes \mathbb{K} \rightarrow 0$ via the coordinate projection $\left(\mathfrak{I}_{1} \oplus \mathfrak{I}_{2}\right) \otimes \mathbb{K} \rightarrow \mathfrak{I}_{i} \otimes \mathbb{K}$ is essential, and hence full since $\mathcal{Q}\left(\mathfrak{I}_{i} \otimes \mathbb{K}\right)$ is simple. Thus, by Lemma 3.6, $0 \rightarrow\left(\mathfrak{I}_{1} \oplus \mathfrak{I}_{2}\right) \otimes \mathbb{K} \rightarrow \mathfrak{I} \otimes \mathbb{K} \rightarrow$ $\mathfrak{I} /\left(\mathfrak{I}_{1} \oplus \mathfrak{I}_{2}\right) \otimes \mathbb{K} \rightarrow 0$ is full. By [ERRa, Proposition 5.4], $0 \rightarrow\left(\mathfrak{I}_{1} \oplus \mathfrak{I}_{2}\right) \otimes \mathbb{K} \rightarrow$ $\mathfrak{A} \otimes \mathbb{K} \rightarrow \mathfrak{A} /\left(\mathfrak{I}_{1} \oplus \mathfrak{I}_{2}\right) \otimes \mathbb{K} \rightarrow 0$ is a full extension since $\mathfrak{I} /\left(\mathfrak{I}_{1} \oplus \mathfrak{I}_{2}\right)$ is an essential ideal of $\mathfrak{A} /\left(\mathfrak{I}_{1} \oplus \mathfrak{I}_{2}\right)$.

Lemma 5.14. Let $\mathfrak{A}$ be a graph $C^{*}$-algebra with tempered signature 4.3E.7. Let $\mathfrak{I}$ be the ideal of $\mathfrak{A}$ such that $\mathfrak{A} / \mathfrak{I}$ is simple. Then $0 \rightarrow \mathfrak{I} \otimes \mathbb{K} \rightarrow \mathfrak{A} \otimes \mathbb{K} \rightarrow \mathfrak{A} / \mathfrak{I} \otimes \mathbb{K} \rightarrow 0$ is a full extension.

Proof. Let $\mathfrak{I}_{1}$ and $\mathfrak{I}_{2}$ be the minimal ideals of $\mathfrak{A}$ which is contained in $\mathfrak{I}$. Since $\mathfrak{I} /\left(\mathfrak{I}_{1}+\mathfrak{I}_{2}\right)$ is a non-unital, purely infinite, simple $C^{*}$-algebra, we have that $0 \rightarrow$ $\mathfrak{I} /\left(\mathfrak{I}_{1}+\mathfrak{I}_{2}\right) \otimes \mathbb{K} \rightarrow \mathfrak{A} /\left(\mathfrak{I}_{1}+\mathfrak{I}_{2}\right) \otimes \mathbb{K} \rightarrow \mathfrak{A} / \mathfrak{I} \otimes \mathbb{K} \rightarrow 0$ is a full extension. The conclusion of the lemma now follows from Corollary 5.3 of [ERRa].

Lemma 5.15. Let $\mathfrak{A}$ be a graph $C^{*}$-algebra with tempered signature 4.1F.E. Let $\mathfrak{I}$ be the smallest ideal of $\mathfrak{A}$. Then $0 \rightarrow \mathfrak{I} \otimes \mathbb{K} \rightarrow \mathfrak{A} \otimes \mathbb{K} \rightarrow \mathfrak{A} / \mathfrak{I} \otimes \mathbb{K} \rightarrow 0$ is a full extension.

Proof. Let $\mathfrak{I}_{1}$ be the ideal of $\mathfrak{A}$ such that $\mathfrak{I}_{1}$ contains $\mathfrak{I}$ and $\mathfrak{I}_{1} / \mathfrak{I}$ is simple. Since $\mathfrak{I}_{1}$ is stably isomorphic to a non- $A F$ graph $C^{*}$-algebra with exactly one nontrivial ideal, we have that $0 \rightarrow \mathfrak{I} \otimes \mathbb{K} \rightarrow \mathfrak{I}_{1} \otimes \mathbb{K} \rightarrow \mathfrak{I}_{1} / \mathfrak{I} \otimes \mathbb{K} \rightarrow 0$ is full. Since $\mathfrak{I}_{1} / \mathfrak{I}$ is an essential ideal of $\mathfrak{A} / \mathfrak{I}$, the conclusion of the lemma follows from Proposition 5.4 of ERRa.

Using the above lemmas and the results of ARR12, we get the following: 
Corollary 5.16. Let $\mathfrak{A}$ and $\mathfrak{B}$ be graph $C^{*}$-algebras with signature either $4.1 F$ or $4.3 E$, and assume that there exists an isomorphism from $\mathrm{FK}_{X}^{+}(\mathfrak{A})$ to $\mathrm{FK}_{X}^{+}(\mathfrak{B})$. If

(1) $\mathfrak{A}$ and $\mathfrak{B}$ both have tempered signature 4.1F.7, 4.1F.E, 4.3E.3, 4.3E.7, or 4.3E.D, or

(2) $\mathfrak{A}$ and $\mathfrak{B}$ both have finitely generated $K$-theory and have tempered signature 4.1F.D, 4.3E.4 or 4.3E.5, or

(3) $\mathfrak{A}$ and $\mathfrak{B}$ both are unital and have tempered signature 4.1F.2, 4.1F.5, 4.1F.6, 4.3E.9 or $4.3 E . B$,

then $\mathfrak{A} \otimes \mathbb{K} \cong \mathfrak{B} \otimes \mathbb{K}$.

Proof. By the above lemmas, all the extensions are full. Note that the specified ideal and quotient for each space belongs to classes of $C^{*}$-algebras satisfying the conditions in Definition 5.2. Hence, the result now follows from Theorem 5.1

\subsection{O-shaped spaces.}

Lemma 5.17. Let $\mathfrak{A}$ be a graph $C^{*}$-algebra that is a tight $C^{*}$-algebra over the $O$ shaped space 4.3B.7. Let $\mathfrak{I}$ be the smallest ideal of $\mathfrak{A}$ and let $\mathfrak{I}_{1}$ and $\mathfrak{I}_{2}$ be the ideals of $\mathfrak{A}$ which contain $\mathfrak{I}$ and $\mathfrak{I}_{k} / \mathfrak{I}$ is simple. Then $0 \rightarrow\left(\mathfrak{I}_{1}+\mathfrak{I}_{2}\right) \otimes \mathbb{K} \rightarrow \mathfrak{A} \otimes \mathbb{K} \rightarrow$ $\mathfrak{A} /\left(\mathfrak{I}_{1}+\mathfrak{I}_{2}\right) \otimes \mathbb{K} \rightarrow 0$ is a full extension.

Proof. Note that $\mathfrak{A} / \mathfrak{I}$ is a tight $C^{*}$-algebra over the space 3.6.5. Then by Lemma3.6. $0 \rightarrow\left(\mathfrak{I}_{1}+\mathfrak{I}_{2}\right) / \mathfrak{I} \otimes \mathbb{K} \rightarrow \mathfrak{A} / \mathfrak{I} \otimes \mathbb{K} \rightarrow \mathfrak{A} /\left(\mathfrak{I}_{1}+\mathfrak{I}_{2}\right) \otimes \mathbb{K} \rightarrow 0$ is a full extension since $\mathfrak{I}_{1} / \mathfrak{I}$ and $\mathfrak{I}_{2} / \mathfrak{I}$ are purely infinite, simple $C^{*}$-algebras. Also, since $\mathfrak{I}$ is an essential ideal of $\mathfrak{I}_{1}+\mathfrak{I}_{2}$ and since $\mathfrak{I}$ is a purely infinite, simple $C^{*}$-algebra, we have that $0 \rightarrow \mathfrak{I} \otimes \mathbb{K} \rightarrow\left(\mathfrak{I}_{1}+\mathfrak{I}_{2}\right) \otimes \mathbb{K} \rightarrow\left(\mathfrak{I}_{1}+\mathfrak{I}_{2}\right) / \mathfrak{I} \otimes \mathbb{K} \rightarrow 0$ is a full extension. The conclusion of the lemma now follows from Proposition 3.2 of ERR10 since $\mathfrak{A} /\left(\mathfrak{I}_{1}+\mathfrak{I}_{2}\right)$ is simple.

Lemma 5.18. Let $\mathfrak{A}$ be a graph $C^{*}$-algebra that is a tight $C^{*}$-algebra over the $O$ shaped space 4.3B.E. Let $\mathfrak{I}$ be the smallest ideal of $\mathfrak{A}$. Then $0 \rightarrow \mathfrak{I} \otimes \mathbb{K} \rightarrow \mathfrak{A} \otimes \mathbb{K} \rightarrow$ $\mathfrak{A} / \mathfrak{I} \otimes \mathbb{K} \rightarrow 0$ is a full extension.

Proof. Let $\mathfrak{I}_{1}$ and $\mathfrak{I}_{2}$ be the ideals of $\mathfrak{A}$ which contain $\mathfrak{I}$ and $\mathfrak{I}_{k} / \mathfrak{I}$ is simple. Since $\mathfrak{I}_{k} \otimes \mathbb{K}$ is isomorphic to a graph $C^{*}$-algebra with exactly one non-trivial ideal and $\mathfrak{I}_{k} \otimes \mathbb{K}$ is not an $A F$ algebra, by Proposition [3.9, we have that $0 \rightarrow \mathfrak{I} \otimes \mathbb{K} \rightarrow$ $\mathfrak{I}_{k} \otimes \mathbb{K} \rightarrow \mathfrak{I}_{k} / \mathfrak{I} \otimes \mathbb{K} \rightarrow 0$ is a full extension. By Lemma 3.14, $0 \rightarrow \mathfrak{I} \otimes \mathbb{K} \rightarrow$ $\left(\mathfrak{I}_{1}+\mathfrak{I}_{2}\right) \otimes \mathbb{K} \rightarrow\left(\mathfrak{I}_{1}+\mathfrak{I}_{2}\right) / \mathfrak{I} \otimes \mathbb{K} \rightarrow 0$ is a full extension. The conclusion of the lemma now follows from Proposition 5.4 of ERR09 since $\left(\mathfrak{I}_{1}+\mathfrak{I}_{2}\right) / \mathfrak{I} \otimes \mathbb{K}$ is an essential ideal of $\mathfrak{A} / \mathfrak{I}$.

Using the above lemmas and the results of [ABK, we get the following cases:

Corollary 5.19. Let $\mathfrak{A}$ and $\mathfrak{B}$ be graph $C^{*}$-algebras that are tight over a $O$-shaped space $X$. Assume that there exists an isomorphism from $\mathrm{FK}_{X}^{+}(\mathfrak{A})$ to $\mathrm{FK}_{X}^{+}(\mathfrak{B})$. If $\mathfrak{A}$ and $\mathfrak{B}$ both have tempered signature $4.3 B .7$ or $4.3 B$.E, then $\mathfrak{A} \otimes \mathbb{K} \cong \mathfrak{B} \otimes \mathbb{K}$.

Proof. By the above lemmas, all the extensions are full. Note that the specified ideal and quotient for each space belongs to classes of $C^{*}$-algebras satisfying the conditions in Definition 5.2. Hence, the result now follows from Theorem 5.1. 


\section{Summary of Results}

In this final section, we index our results. Cases that open are indicated by "?". Cases that are solved in general are marked by " $\sqrt{ } "$, and if we need to impose conditions of finitely generated $K$-theory or unitality, this is indicated by " $\sqrt{f}_{f . g}$." or " $\sqrt{1}$ ", respectively.

6.1. One point spaces. Having nothing new to add, we include the simple case only for completeness.

\begin{tabular}{|l|l|l|l|}
\hline \multicolumn{4}{|c|}{ 1.0.x } \\
\hline \hline 0 & $\square$ & $\sqrt{ }$ & Theorem 2.1 \\
\hline 1 & $\mathbf{\square}$ & $\sqrt{ }$ & Theorem 2.3 \\
\hline
\end{tabular}

6.2. Two point spaces. This case was solved in [ET10, so again we include it only for completeness.

\begin{tabular}{|c|c|c|c|}
\hline \multicolumn{3}{|c|}{ 2.1.x } \\
\hline \hline 0 & $\square \longrightarrow \square$ & $\sqrt{ }$ & Theorem 2.1 \\
\hline 1 & $\square \longrightarrow \square$ & $\sqrt{ }$ & Proposition 2.5 \\
\hline 2 & $\mathbf{\square} \square$ & $\sqrt{ }$ & Proposition 2.6 \\
\hline 3 & $\mathbf{\square}$ & $\sqrt{ }$ & Theorem 2.3 \\
\hline
\end{tabular}

6.3. Three point spaces. We resolve the case of three primitive ideal spaces here, up to a condition of finite generation which must be imposed in the cases of signature 3.7.2 and 3.7.5. We do not know if this condition is necessary.

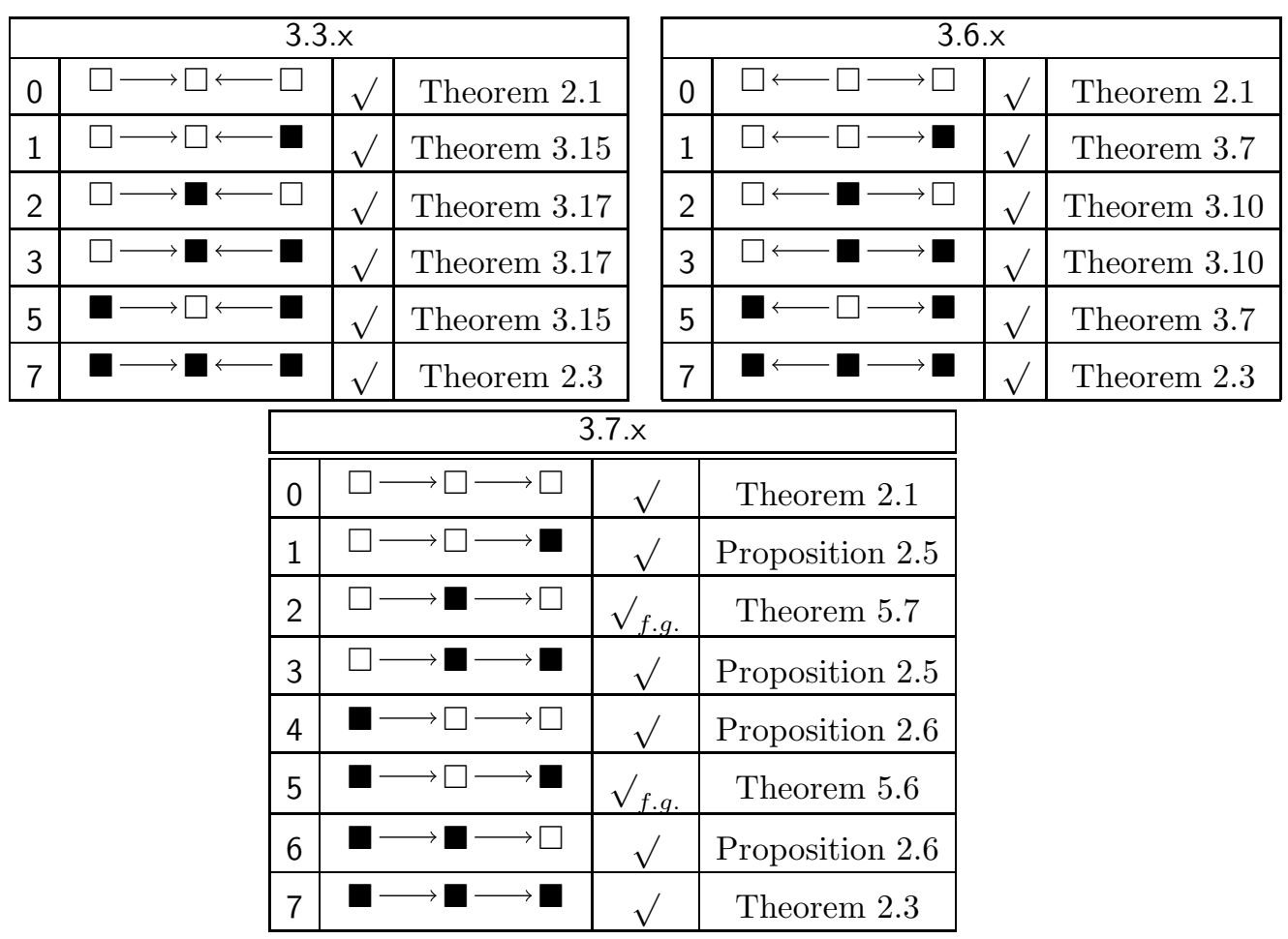


6.4. Four point spaces. In this section, we present our results for the case of four primitive ideals. As will be obvious below, the strength of our results varies dramatically with the nature of the spaces. In general, we can say quite a lot about all spaces apart from 4.E, 4.1E, and 4.3B. It may be interesting to note what makes these spaces difficult to handle; indeed the case 4.E is an accordion space in which a general UCT is know to hold, but it differs from the other accordion spaces by having poor separation properties when it comes to establishing fullness. The $O$ shaped spaces are also hard to separate fully, but have the added difficulty that no general UCT is known for them. 


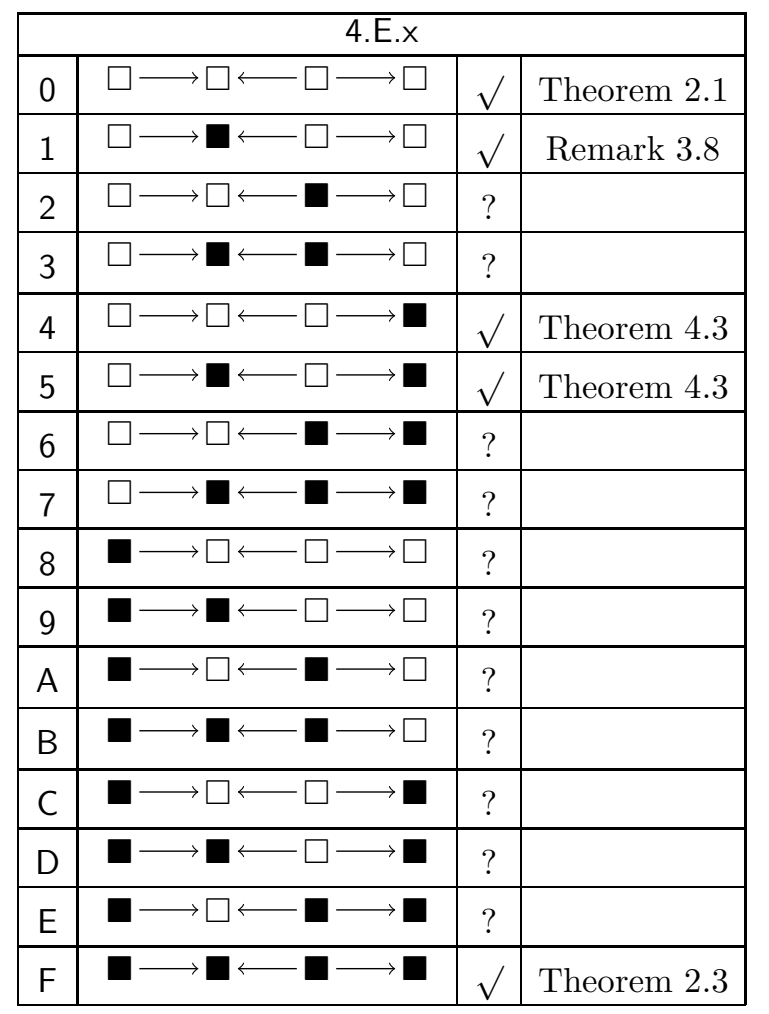

\begin{tabular}{|c|c|c|c|}
\hline \multicolumn{4}{|c|}{ 4.F.x } \\
\hline 0 & $\overrightarrow{\square \longrightarrow \square \longleftarrow \square \longleftarrow \square}$ & $\sqrt{ }$ & Theorem 2.1 \\
\hline 1 & $\square \longrightarrow \boldsymbol{\square} \longleftarrow \square \longleftarrow \square$ & $\sqrt{ }$ & Proposition 2.5 \\
\hline 2 & $\square \longrightarrow \square \longleftarrow \mathbf{\square} \longleftarrow \square$ & $\sqrt{1}$ & Corollary 5.11 \\
\hline 3 & $\square \longrightarrow \mathbf{\square} \longleftarrow \mathbf{\square} \longleftarrow \square$ & $\sqrt{f . g .}$ & Corollary 5.11 \\
\hline 4 & $\square \longrightarrow \square \longleftarrow \square \longleftarrow \mathbf{\square}$ & $\sqrt{ } 1$ & Corollary 5.11 \\
\hline 5 & $\square \longrightarrow \boldsymbol{\square} \longleftarrow \square \longleftarrow \mathbf{\square}$ & $\sqrt{ } 1$ & Corollary 5.11 \\
\hline 6 & $\square \longrightarrow \square \longleftarrow \mathbf{\square} \longleftarrow \mathbf{\square}$ & $\sqrt{ }$ & Theorem 3.15 \\
\hline 7 & $\square \longrightarrow \mathbf{\square} \longleftarrow \mathbf{D} \longleftarrow \mathbf{\square}$ & $\sqrt{ }$ & Corollary 5.11 \\
\hline 8 & $\mathbf{\square} \longrightarrow \square \longleftarrow \square \longleftarrow \square$ & $\sqrt{ }$ & Theorem 3.15 \\
\hline 9 & $\boldsymbol{\mathbf { a }} \longrightarrow \boldsymbol{\square} \longleftarrow \square \longleftarrow \square$ & $\sqrt{ }$ & Corollary 5.11 \\
\hline A & 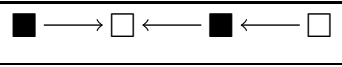 & $\sqrt{f . g .}$ & Corollary 5.11 \\
\hline B & $\mathbf{\square} \longrightarrow \boldsymbol{\square} \longleftarrow \mathbf{\square} \longleftarrow \square$ & $\sqrt{f . g .}$ & Corollary 5.11 \\
\hline C & $\mathbf{D} \longrightarrow \square \longleftarrow \square \longleftarrow \mathbf{\square}$ & $\sqrt{1}$ & Corollary 5.11 \\
\hline $\mathrm{D}$ & $\mathbf{\square} \longrightarrow \mathbf{\square} \square \longleftarrow \mathbf{\square}$ & $\sqrt{1}$ & Corollary 5.11 \\
\hline$E$ & $\mathbf{\square} \longrightarrow \square \longleftarrow \mathbf{\square} \mathbf{\square}$ & $\sqrt{ }$ & Theorem 3.15 \\
\hline $\mathrm{F}$ & $\mathbf{\square} \longrightarrow \mathbf{\square} \longleftarrow \mathbf{\square} \longleftarrow \mathbf{\square}$ & $\sqrt{ }$ & Theorem 2.3 \\
\hline
\end{tabular}




\begin{tabular}{|c|c|c|c|}
\hline \multicolumn{4}{|c|}{$4.39 . x$} \\
\hline 0 & $\square \longleftarrow \square \longrightarrow \square \longrightarrow \square$ & $\sqrt{ }$ & Theorem 2.1 \\
\hline 1 & $\square \longleftarrow \square \longrightarrow \square \longrightarrow \mathbf{\square}$ & $\sqrt{ }$ & Theorem 3.7 \\
\hline 2 & $\square \longleftarrow \square \longrightarrow \mathbf{\square} \longrightarrow \square$ & $\sqrt{ } 1$ & Corollary 5.11 \\
\hline 3 & $\square \longleftarrow \square \longrightarrow \mathbf{\square} \longrightarrow \boldsymbol{\square}$ & $\sqrt{ }$ & Theorem 3.7 \\
\hline 4 & $\mathbf{\mathbf { a }} \square \longrightarrow \square \longrightarrow \square$ & $\sqrt{ }$ & Theorem 3.7 \\
\hline 5 & 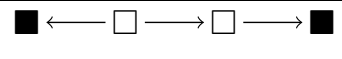 & $\sqrt{ }$ & Theorem 3.7 \\
\hline 6 & $\mathbf{\square} \longleftarrow \square \longrightarrow \mathbf{D} \longrightarrow \square$ & $\sqrt{ }_{1}$ & Corollary 5.11 \\
\hline 7 & $\mathbf{\mathbf { a }} \square \longrightarrow \mathbf{\square} \longrightarrow \mathbf{\square}$ & $\sqrt{ }$ & Theorem 3.7 \\
\hline 8 & $\square \longleftarrow \mathbf{\square} \longrightarrow \square \longrightarrow \square$ & $\sqrt{ }$ & Proposition 2.6 \\
\hline 9 & $\square \longleftarrow \mathbf{\square} \longrightarrow \square \longrightarrow \boldsymbol{\square}$ & $\sqrt{ }_{f, a}$ & Corollary 5.11 \\
\hline A & $\square \longleftarrow \mathbf{\square} \longrightarrow \mathbf{D} \longrightarrow \square$ & $\sqrt{1}$ & Corollary 5.11 \\
\hline B & $\square \longleftarrow \mathbf{\square} \longrightarrow \mathbf{\square} \longrightarrow \mathbf{\square}$ & $\sqrt{ }$ & Corollary 5.11 \\
\hline C & $\mathbf{\mathbf { a }} \mathbf{\mathbf { a }} \longrightarrow \square \longrightarrow \square$ & $\sqrt{ }$ & Corollary 5.11 \\
\hline D & $\mathbf{\square} \longleftarrow \mathbf{\square} \longrightarrow \square \longrightarrow \mathbf{\square}$ & $\sqrt{f f . g}$ & Corollary 5.11 \\
\hline $\mathrm{E}$ & $\mathbf{\mathbf { a }} \longleftarrow \mathbf{\square} \longrightarrow \mathbf{\square} \longrightarrow \square$ & $\sqrt{ } 1$ & Corollary 5.11 \\
\hline $\mathrm{F}$ & $\mathbf{a} \longleftarrow \mathbf{a} \longrightarrow \mathbf{a} \longrightarrow \mathbf{\square}$ & $\sqrt{ }$ & Theorem 2.3 \\
\hline
\end{tabular}

\begin{tabular}{|c|c|c|c|}
\hline \multicolumn{4}{|c|}{$4.3 \mathrm{~F} . \mathrm{x}$} \\
\hline 0 & $\overrightarrow{\square \longrightarrow \square \longrightarrow \square \longrightarrow \square}$ & $\sqrt{ }$ & Theorem 2.1 \\
\hline 1 & $\square \longrightarrow \square \longrightarrow \square \longrightarrow \boldsymbol{\square}$ & $\sqrt{ }$ & Proposition 2.5 \\
\hline 2 & $\square \longrightarrow \square \longrightarrow \mathbf{\square} \longrightarrow \square$ & $\sqrt{f . g .}$ & Theorem 5.7 \\
\hline 3 & $\overrightarrow{\square \longrightarrow \square \longrightarrow \mathbf{D} \longrightarrow \mathbf{\square}}$ & $\sqrt{ }$ & Proposition 2.5 \\
\hline 4 & 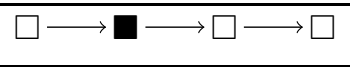 & $\sqrt{f . g .}$ & Theorem 5.7 \\
\hline 5 & $\square \longrightarrow \boldsymbol{\square} \longrightarrow \square \longrightarrow \boldsymbol{\square}$ & $\sqrt{f . g .}$ & Corollary 5.11 \\
\hline 6 & 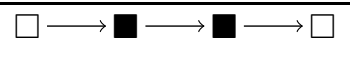 & $\sqrt{1}$ & Corollary 5.11 \\
\hline 7 & $\square \longrightarrow \mathbf{\square} \longrightarrow \mathbf{\square} \longrightarrow \mathbf{\square}$ & $\sqrt{ }$ & Proposition 2.5 \\
\hline 8 & $\mathbf{\square} \longrightarrow \square \longrightarrow \square \longrightarrow \square$ & $\sqrt{ }$ & Proposition 2.6 \\
\hline 9 & $\mathbf{\square} \longrightarrow \square \longrightarrow \square \longrightarrow \boldsymbol{\square}$ & $\sqrt{f . g .}$ & Theorem 5.6 \\
\hline$A$ & $\mathbf{\square} \longrightarrow \square \longrightarrow \mathbf{D} \longrightarrow \square$ & $\sqrt{1}$ & Corollary 5.11 \\
\hline $\bar{B}$ & $\mathbf{\square} \longrightarrow \square \longrightarrow \mathbf{D} \longrightarrow \boldsymbol{\square}$ & $?$ & \\
\hline$C$ & $\mathbf{\square} \longrightarrow \mathbf{n} \longrightarrow \square \longrightarrow \square$ & $\sqrt{ }$ & Proposition 2.6 \\
\hline $\mathrm{D}$ & $\mathbf{\square} \longrightarrow \boldsymbol{\square} \longrightarrow \square \longrightarrow \mathbf{\square}$ & $\sqrt{f . g .}$ & Corollary 5.11 \\
\hline$E$ & $\boldsymbol{\nabla} \longrightarrow \boldsymbol{\square} \longrightarrow \boldsymbol{\square} \longrightarrow \square$ & $\sqrt{ }$ & Proposition 2.6 \\
\hline $\mathrm{F}$ & $\mathbf{\square} \longrightarrow \mathbf{\square} \longrightarrow \mathbf{\square} \longrightarrow \mathbf{\square}$ & $\sqrt{ }$ & Theorem 2.3 \\
\hline
\end{tabular}



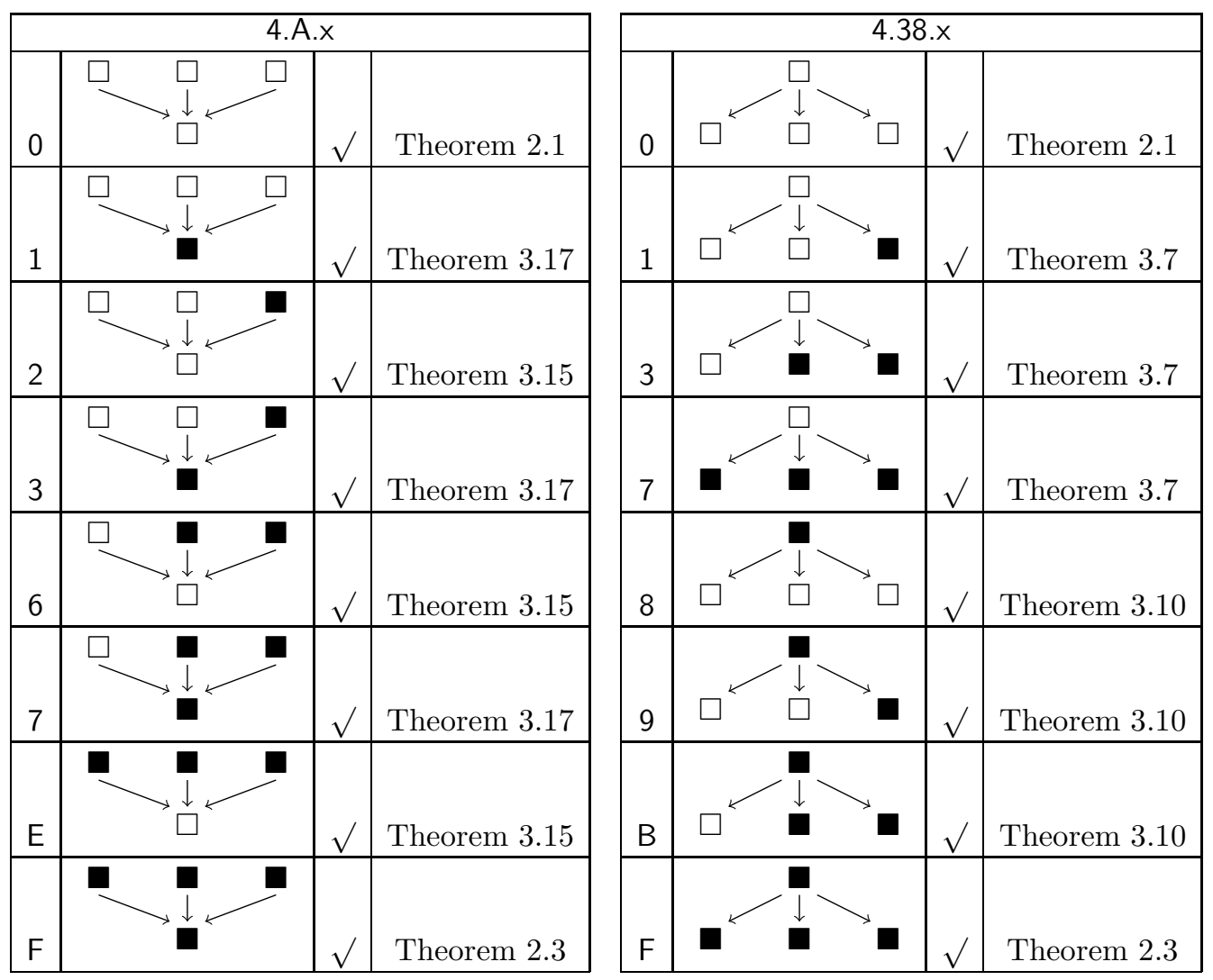


\begin{tabular}{|c|c|c|c|}
\hline \multicolumn{4}{|c|}{$4.1 \mathrm{~F} . \mathrm{x}$} \\
\hline 0 & $\begin{array}{l}\square \square \\
\downarrow \\
\square \rightarrow \square\end{array}$ & $\sqrt{ }$ & Theorem 2.1 \\
\hline 1 & $\square$ & $\sqrt{ }$ & Proposition 2.5 \\
\hline 2 & & $\sqrt{ } 1$ & Corollary 5.16 \\
\hline 3 & & $\sqrt{ }$ & Proposition 2.5 \\
\hline 4 & & $\sqrt{ }$ & Remark 3.16 \\
\hline 5 & & $\sqrt{1}$ & Corollary 5.16 \\
\hline 6 & & $\sqrt{ }{ }_{1}$ & Corollary 5.16 \\
\hline 7 & & $\sqrt{ }$ & Corollary 5.16 \\
\hline C & & $\sqrt{ }$ & Remark 3.16 \\
\hline D & & $\sqrt{f . g .}$ & Corollary 5.16 \\
\hline$E$ & & $\sqrt{ }$ & Corollary 5.16 \\
\hline $\mathrm{F}$ & & $\sqrt{ }$ & Theorem 2.3 \\
\hline
\end{tabular}

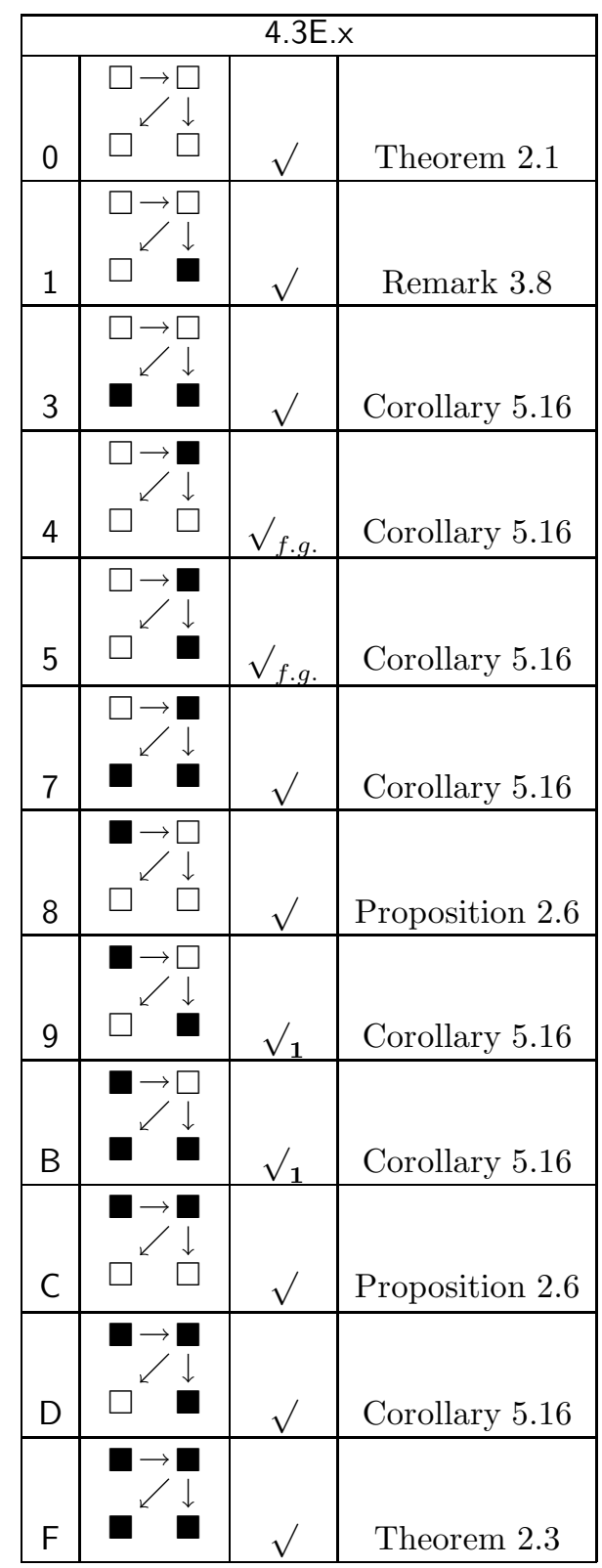




\begin{tabular}{|c|c|c|c|}
\hline \multicolumn{4}{|c|}{$4.1 \mathrm{E} . \mathrm{x}$} \\
\hline 0 & $\square \leftarrow \square$ & $\sqrt{ }$ & Theorem 2.1 \\
\hline 1 & & $\sqrt{ }$ & Remark 3.8 \\
\hline 3 & & $\sqrt{ }$ & Remark 3.8 \\
\hline 4 & $\begin{array}{l}\square \rightarrow \square \\
\downarrow \\
\square \leftarrow \mathbf{\square}\end{array}$ & $\sqrt{ }$ & Remark 3.16 \\
\hline 5 & $\begin{array}{l}\square \rightarrow \square \\
\downarrow \\
\downarrow \\
\mathbf{\square}\end{array}$ & $?$ & \\
\hline 7 & $\begin{array}{l}\square \rightarrow \mathbf{\square} \\
\downarrow \\
\mathbf{a} \\
\end{array}$ & $?$ & \\
\hline C & $\begin{array}{l}\mathbf{\square} \rightarrow \square \\
\downarrow \\
\square \leftarrow \mathbf{\square}\end{array}$ & $\sqrt{ }$ & Remark 3.16 \\
\hline D & $\stackrel{\downarrow}{\mathbf{a}} \leftarrow \mathbf{a}$ & $?$ & \\
\hline $\mathrm{F}$ & $\boldsymbol{D} \leftarrow \mathbf{\square}$ & $?$ & \\
\hline
\end{tabular}

\begin{tabular}{|c|c|c|c|}
\hline \multicolumn{4}{|c|}{$4.3 \mathrm{~B} . \mathrm{x}$} \\
\hline 0 & $\square \rightarrow \square^{v}$ & $\sqrt{ }$ & Theorem 2.1 \\
\hline 1 & $\begin{array}{l}\square \rightarrow \square \\
\downarrow \\
\square \rightarrow \downarrow\end{array}$ & $\sqrt{ }$ & Proposition 2.5 \\
\hline 2 & $\begin{array}{l}\square \rightarrow \square \\
\downarrow \\
\downarrow \\
\square\end{array}$ & $?$ & \\
\hline 3 & $\begin{array}{l}\square \rightarrow \square \\
\downarrow \\
\downarrow \\
\square\end{array}$ & $?$ & \\
\hline 6 & $\begin{array}{l}\square \rightarrow \square \\
\downarrow \\
\downarrow \\
\square\end{array}$ & $?$ & \\
\hline 7 & $\begin{array}{l}\square \rightarrow \boldsymbol{\square} \\
\downarrow \\
\boldsymbol{\square} \\
\rightarrow\end{array}$ & $\sqrt{ }$ & Corollary 5.19 \\
\hline 8 & $\square-$ & $\sqrt{ }$ & Proposition 2.6 \\
\hline 9 & $\stackrel{\downarrow}{\square} \rightarrow 1$ & $?$ & \\
\hline A & $\mathbf{n} \rightarrow \square$ & $?$ & \\
\hline B & $\mathbf{\square} \rightarrow$ & ? & \\
\hline$E$ & $\underset{\mathbf{a}}{\downarrow} \rightarrow$ & $\sqrt{ }$ & Corollary 5.19 \\
\hline $\mathrm{F}$ & $\boldsymbol{v} \rightarrow$ & $\sqrt{ }$ & Theorem 2.3 \\
\hline
\end{tabular}




\section{REFERENCES}

[ABK] S. Arklint, R. Bentmann, and T. Katsura, Reduction of filtered K-theory and a characterization of Cuntz-Krieger algebras, in preparation.

[ARR12] S. Arklint, G. Restorff, and E. Ruiz, Filtrated $K$-theory for real rank zero $C^{*}$-algebras, Int. J. Math. 23 (2012).

[BHRS] T. Bates, J. Hong, I. Raeburn, and W. Szymański, The ideal structure of the $C^{*}$-algebras of infinite graphs, Illinois J. Math., 46 (2002), 1159-1176.

[BK] R. Bentmann and M. Köhler, Universal coefficient theorems for $C^{*}$-algebras over finite topological spaces, preprint arXiv:1101.5702

[BB] B. Blackadar, Operator algebras, Springer-Verlag, Berlin, 2006.

[BM02] G. Brinkmann and B.D. McKay, Posets on up to 16 points, Order 19 (2002), no. 2, $147-179$.

[Bro77] L.G. Brown, Stable isomorphism of hereditary subalgebras of $C^{*}$-algebras, Pacific J. Math. 71 (1977), no. 2, 335-348.

[CET] T.M. Carlsen, S. Eilers, and M. Tomforde, Index maps in the K-theory of graph algebras, J. K-Theory 9 (2012), 385-406.

[DT02] D. Drinen and M. Tomforde, Computing $K$-theory and Ext for graph $C^{*}$-algebras, Illinois J. Math.46 (2002), 81-91.

[DT05] D. Drinen and M. Tomforde, The $C^{*}$-algebras of arbitrary graphs, Rocky Mountain J. Math. 35 (2005), 105-135.

[EK] S. Eilers and T. Katsura, Semiprojectivity and properly infinite projections in graph $C^{*}$-algebra, in preparation.

[EK01] G.A. Elliott and D. Kucerovsky, An abstract Voiculescu-Brown-Douglas-Fillmore absorption theorem, Pacific J. Math. 198 (2001), no. 2, 385-409.

[Ell76] G.A. Elliott, On the classification of inductive limits of sequences of semisimple finitedimensional algebras, J. Algebra 38 (1976), no. 1, 29-44.

[Ell10] - Towards a theory of classification, Adv. Math. 223 (2010), no. 1, 30-48.

[ELP99] S. Eilers, T.A. Loring, and G.K. Pedersen, Morphisms of extensions of $C^{*}$-algebras: pushing forward the Busby invariant, Adv. Math. 147 (1999), no. 1, 74-109.

[ERRa] S. Eilers, G. Restorff, and E. Ruiz, Classifying $C^{*}$-algebras with both finite and infinite subquotients, preprint arXiv:1009.4778

[ERRb] - The ordered $K$-theory of a full extension, preprint arXiv: 1106.1551.

[ERRc] Strong classification of extensions of classifiable $C^{*}$-algebras, in preparation.

[ERR09] _ Classification of extensions of classifiable $C^{*}$-algebras, Adv. Math. 222 (2009), $2153-2172$.

[ERR10] - On graph $C^{*}$-algebras with a linear ideal lattice, Bull. Malays. Math. Sci. Soc. 33 (2010), no. 2, 233-241.

[ERS11] S. Eilers, E. Ruiz, and A.P.W. Sørensen, Amplified graph $C^{*}$-algebras, to appear in Münster J. Math, 2011.

[ET10] S. Eilers and M. Tomforde, On the classification of nonsimple graph algebras, Math. Ann. 346 (2010), 393-418.

[JJ] JA. Jeong, Real rank of $C^{*}$-algebras associated with graphs, J. Aust. Math. Soc., 77 (2004), 141-147.

[Kir94] E. Kirchberg, The classification of purely infinite $C^{*}$-algebras using Kasparov's theory, preprint, third draft, 1994.

[Kir00] E. Kirchberg, Das nicht-kommutative Michael-Auswahlprinzip und die Klassifikation nicht-einfacher Algebren, $C^{*}$-algebras (Münster, 1999), Springer, Berlin, 2000, pp. 92141.

[KR00] E. Kirchberg and M. Rørdam, Non-simple purely infinite $C^{*}$-algebras, Amer. J. Math., 122 (2000), 637-666.

[KR02] E. Kirchberg and M. Rørdam, Infinite non-simple $C^{*}$-algebras: absorbing the Cuntz algebras $\mathcal{O}_{\infty}$, Adv. Math., 167 (2002), 195-264.

[KN06] D. Kucerovsky and P. W. Ng, The corona factorization property and approximate unitary equivalence, Houston J. Math. 32 (2006), no. 2, 531-550 (electronic).

[MNa] R. Meyer and R. Nest, $C^{*}$-algebras over topological spaces: Filtrated $K$-theory, Canad. J. Math. 64 (2012), no. 2, 368-408. 
[MNb] $-C^{*}$-algebras over topological spaces: The bootstrap class, Münster J. Math. 2 (2009), 215-252.

[Mur90] G.J. Murphy, $C^{*}$-algebras and operator theory, Academic Press, San Diego, 1990.

[Ped99] G.K. Pedersen, Pullback and pushout constructions in $C^{*}$-algebra theory, J. Funct. Anal. 167 (1999), 243-344.

[NCP00] N.C. Phillips, A classification theorem for nuclear purely infinite simple $C^{*}$-algebras, Doc. Math. 5 (2000), 49-114.

[Rae05] I. Raeburn, Graph algebras, CBMS Regional Conference Series in Mathematics, vol. 103, Published for the Conference Board of the Mathematical Sciences, Washington, DC, 2005.

[Res08] G. Restorff, Classification of non-simple $C^{*}$-algebras, Ph.D. thesis, Department of Mathematical Sciences, University of Copenhagen, 2008, http://www.math.ku.dk/ restorff/papers/thesis.pdf

[Ror97] M. Rørdam, Classification of extensions of certain $C^{*}$-algebras by their six term exact sequences in K-theory, Math. Ann. 308 (1997), no. 1, 93-117.

[MT07] M. Tomforde, Uniqueness theorems and ideal structure for Leavitt path algebras, J. Algebra, 318 (2007), 270-299.

[TW07] A. Toms and W. Winter, Strongly self-absorbing $C^{*}$-algebras, Trans. Amer. Math. Soc., 359 (2007), 3999-4029.

[Zha91] S. Zhang, K1-groups, quasidiagonality, and interpolation by multiplier projections, Trans. Amer. Math. Soc. 325 (1991), no. 2, 793-818.

Department of Mathematical Sciences, University of Copenhagen, Universitetsparken 5, DK-2100 Copenhagen, Denmark

E-mail address: eilers@math.ku.dk

Department of Science and Technology, University of the Faroe Islands, Nóatún 3, FO-100 Tórshavn, Faroe Islands

E-mail address: gunnarr@setur.fo

Department of Mathematics, University of Hawail, Hilo, 200 W. Kawili St., Hilo, HAWAII, 96720-4091 USA

E-mail address: ruize@hawaii.edu 